\title{
Production of bioactive metabolites from different marine endophytic Streptomyces species and testing them against methicillin-resistant Staphylococcus aureus (MRSA) and cancer cell lines
}

\author{
Mervat Morsy Abbas Ahmed El-Gendy ${ }^{1,2 *}$, Zeinat K. Mohamed ${ }^{3}$, NAHEd Z. HeKAL $^{3}$, \\ FATEN M. ALI ${ }^{4}$, AMIRA E. M. YOUSEF ${ }^{3}$ \\ ${ }^{1}$ Department of Biological Sciences, Faculty of Science, University of Jeddah, Jeddah 80203, Saudi Arabia \\ ${ }^{2}$ Chemistry of Natural and Microbial Products Department, National Research Centre, Dokki, Giza, Egypt \\ ${ }^{3}$ Plant Department, Faculty of Science, Cairo University, Egypt \\ ${ }^{4}$ Microbiology and Immunology Department, Faculty of Medicine, Ain Shams University, Egypt
}

\begin{abstract}
The aim of the work was to identify Actinomycetes strains that are able to produce high levels of anti-MRSA metabolites with a potential use in curing resistant Staphylococcus aureus infections. Twenty-six endophytic Actinomycetes strains were isolated from various marine invertebrates living in two different marine ecosystems (the Mediterranean Sea and the Red Sea). Among them, Streptomyces sp. RS-9 (obtained from the soft coral Sarcophyton sp. from the Red Sea) and Streptomyces sp. MS-26 (obtained from the jellyfish derived from the Mediterranean Sea) demonstrated the highest antagonistic activity against 18 isolates of methicillin-resistant Staphylococcus aureus (MRSA) and 2 isolates of methicillin-resistant coagulase-negative Staphylococci (MRCoNS). The optimization of culture conditions, which includes the time course (at the $6^{\text {th }}$ and the $3^{\text {rd }}$ day of growth), the incubation temperature $\left(27.5^{\circ} \mathrm{C}\right), \mathrm{pH}(8.0$ and 6.0$)$, nutritional requirements comprising different metals, carbon (glucose and glycerol, respectively), and nitrogen (mixture of valine with peanut and a mixture of soybean with methionine and asparagines, respectively) sources for RS-9 and MS-26 strains, respectively, resulted in a 2.2-fold and 2.5-fold increase in the productivity of anti-MRSA metabolites. The GC/MS analysis proved the presence of different bioactive compounds in Streptomyces sp. RS-9 and Streptomyces sp. MS-26 extracts. Two pure compounds, A) 1-Methyl-2-acetyl-6-acetoxy-7-methoxy-(1,2,3,4-tetrahydroisoquinoline) and B) -4-(dimethylamino)1,4,4d,5,12,12a-hexahydro-3,10,11,12a-tetrahydroxy-6-methyl-1,12-dioxo-,[4S-(4- $\alpha, 4 \mathrm{a}$,al]-(2-naphthacene carboxamide, which is a well-known antibiotic called anhydrotetracycline), were isolated from Streptomyces sp. MS-26. Those compounds possessed potent anti-MRSA activity against all the tested clinical MRSA and MRCoNS isolates. The minimum inhibition concentration (MIC) of the above two compounds ranged from 16 to $1024 \mu \mathrm{gml}^{-1}$ and 4 to $128 \mu \mathrm{gml}^{-1}$, respectively. The minimum bactericidal concentration (MBC) ranged from 16 to $1024 \mu^{-1} \mathrm{gml}^{-1}$ and 8 to $128 \mu \mathrm{gml}^{-1}$, respectively with anticancer activity against colon cancer (HCT-16), liver cancer (HepG-2), and lung cancer (A-549) cell lines.
\end{abstract}

Key words : marine endophytic Streptomyces, methicillin-resistant Staphylococcus aureus (MRSA), anti-MRSA, bioactive compounds, anticancer activity

\section{Introduction}

In recent years, Staphylococcus aureus has been one of the main infectious agents causing various diseases extending from relatively benign skin infections to serious, potentially life threatening diseases such as bone and soft tissue intra surgical infections, sepsis, or in-

\footnotetext{
* Corresponding author: ${ }^{1}$ Department of Biological Sciences, Faculty of Science, University of Jeddah, Jeddah 80203, Saudi Arabia;

${ }^{2}$ Chemistry of Natural and Microbial Products Department, National Research Centre, Dokki, Giza, Egypt; e-mail: m_morsy_70@yahoo.com
} 
vasive endocarditis (Awadalla et al., 2010; Lairrull et al., 2009). It is one of the most common causes of both endemic nosocomial infections and the epidemic hospital-acquired infections (Padalkar and Peshwe, 2013). The methicillin-resistant Staphylococcus aureus (MRSA) is a strain that has developed antibiotic resistance to all penicillins, containing methicillin and other $\beta$-lactam antibiotics that limit the possibilities of effective treatment and leave vancomycin as the last therapeutic choice. Unfortunately, recently, vancomycin-resistant strains have also been increasingly detected worldwide (Awadalla et al., 2010; Lairrull et al., 2009). As a result, no antibiotic class is effective against multidrug resistant $S$. aureus infections (Lairrull et al., 2009). Consequently, a discovery of alternative, natural, effective, cheap, and safe antibiotics or chemotherapeutic strategies against this resistant bacterium is necessary.

Marine animals and plants such as sponges, sea squirts, corals, worms, and algae host diverse and abundant endophytic microorganisms capable of biosynthesizing bioactive products (El-Bondkly et al., 2012a; Li, 2009; Ahmed et al., 2015). The marine endophytic Actinomycetes are recognized as an emerging source of natural compounds, which possess unique structures and high activities as antimicrobial and anticancer agents that could be used in medicine and biotechnology $(\mathrm{Li}$, 2009; El-Bondkly et al., 2012b).

This study was aimed at screening and evaluating marine endophytic Actinomycetes of different marine ecosystems (the Red Sea and the Mediterranean Sea) for the production of potent, biologically active substances against clinical methicillin-resistant Staphylococcus aureus (MRSA) and methicillin-resistant coagulase-negative Staphylococci(MRCoNS) isolates. Improvement in the productivity level of bioactive metabolites has been obtained by optimizing cultural and nutritional requirements of selected endophytic strains. Isolation and characterization of these bioactive compounds constituting the determination of their various activities against MRSA, MRCoNS, and different cancer cell lines (colon, liver, and lung cancers) have also been performed.

\section{Materials and methods}

\section{Clinical samples}

Forty one clinical specimens of different cultures (12 sputum, 15 wound, 3 urine, 3 blood, and 8 cultures in- cluding breast discharge, corneal swab, throat swab, synovial fluid, and ear discharge) were collected from patients aged between 1 and over 70 (19 females and 22 males) at El-Demerdash and Ain Shams Specialized hospitals during the period of November 2008 to February 2009. The samples were transported to the laboratory and processed immediately. From these cultures, 18 MRSA and 2 CoNS isolates that showed complete resistance to methicillin and intermediate resistance to vancomycin were obtained and analyzed.

\section{Isolation and identification of clinical multidrug resistant $S$. aureus}

Staphylococcus isolates were isolated and identified using methods reported in Disalvo (1958), Bergey's Manual of Systematic Bacteriology by Williams et al. (1989), Collee and coworkers $(1989,1996)$, Kloos and Lambe (1991), Hass and Defago (2005), and Cherkaoui and coworkers (2007).

\section{Antibiotic sensitivity profile of clinical MRSA isolates}

$S$. aureus isolates were examined for their susceptibility to different types of antibiotics using antibiotic sensitivity disks (Oxoid Limited, England) for glycopeptides (vancomycin), cephalosporins (cefoxitin, cefotaxime, and cefazoline), tetracyclines (doxycycline and oxytetracycline), amino glycosides (amikacin), fluoroquinolones (ciprofloxacin and ofloxacin), $\beta$-lactams (tienam), macrolides (erythromycin), chloramphenicols (chloramphenicol), nitrofurantoin and sulfonamides/trimethoprim (sulfamethazole/trimethoprim) using disk diffusion test (Cheesbrough, 2000). The interpretation of the sensitivity results was performed according to the Clinical \& Laboratory Standards Institute (CLSI), 2009.

\section{Marine invertebrate samples}

Healthy specimens of Egyptian soft coral Sarcophyton sp. and unidentified jellyfish were obtained from the Red Sea (Hurghada) and the Mediterranean Sea (Sidi Bishr shore), respectively. They were transported under cooling conditions and kept at $4{ }^{\circ} \mathrm{C}$ until processing.

\section{Isolation of marine endophytic Actinomycetes}

The endophytic Actinomycete isolates were separated from the inner healthy tissue of each invertebrate according to the procedure described by Taechowisan and coworkers (2003) with slight modifications. The samples were washed with distilled water and cut into 
small pieces of $4 \times 4 \mathrm{~mm}^{2}$. Tissue pieces were rinsed in $0.1 \%$ Tween 20 for $1 \mathrm{~min}$, then in $2.5 \%$ sodium hypochlorite for $15 \mathrm{~min}$, followed by washing in sterile distilled water for $5 \mathrm{~min}$. The surfaces were sterilized with $75 \%$ ethanol for $5 \mathrm{~min}$, and then rinsed 3 times in sterile water. Finally the pieces were plated on inorganic salts starch agar medium Petri dishes containing $100 \mu \mathrm{gml}^{-1}$ nystatin and cycloheximide. Segments of the same origin without surface sterilization were cultured as negative controls to check the presence of contaminated microbes on the tissue segment surfaces. Plates were incubated at $28^{\circ} \mathrm{C}$ for 21 days and Streptomyces single colonies were distinguished, based on their characteristics (tough leathery colonies, branching vegetative mycelia with aerial mycelia, and spore formation).

\section{Detection of antimicrobial activity of marine endophytic} Streptomycetes against clinical MRSA isolates

Muller Hinton (MH) agar media in Petri dishes were seeded with the clinical MRSA or vancomycin intermediate CoNS isolates. They were plated separately using paper assay disks saturated with $30 \mu \mathrm{l}$ of Streptomycete supernatant. Antimicrobial activities of each Streptomycete isolate were assessed against the 18 MRSA isolates using an agar diffusion method (Selva et al., 1996), by measuring the diameter of the inhibition zones (in $\mathrm{mm}$ ). If the inhibition zone reached $10 \mathrm{~mm}, 10-15 \mathrm{~mm}$, and $16-20 \mathrm{~mm}$, the antimicrobial activities were considered to be weakly active $(+)$, active $(++)$, and highly active $(+++)$, respectively. Among Staphylococcal isolates obtained in this study, 2 isolates of coagulase-negative Staphylococci (CoNS D-15 and CoNS-AS15) were used to evaluate the potency of the obtained endophytic Actinomycetes and their extracts, or pure compounds against clinical strains with complete resistance toward methicillin or intermediate resistance against vancomycin.

\section{Phenotypic and chemotypic properties of the selected Actinomycetes (RS-9 and MS-26)}

Based on the analysis of phenotypic and chemotypic characteristics, RS-9 and MS-26 strains were identified. The cover slip technique was used for observing the hyphae and spore chain characters through a light microscope. Spore morphology was studied by examining gold-coated dehydrated specimens with an electron microscope (Kawato and Shinobu, 1959; Zhou et al., 1998). Streptomyces isolates (RS-9 and MS-26) were examined for a broad range of cultural, physiological, and bio- chemical characteristics as described by Szabo and coworkers (1975), Williams and coworkers (1983, 1989), Kämpfer and coworkers (1991), and the International Streptomyces Project (ISP) Scheme as described by Shirling and Gottlieb (1966). The isomers of diaminopimelic acid (DAP) and whole-organism sugars were analyzed following the procedures developed by Hasegawa and coworkers (1983). Menaquinones and phospholipids were performed according to Lechevalier and coworkers (1977) and Minnikin and coworkers (1977). The fatty acid profile was analyzed according to Butte (1983). The base composition of genomic DNA was determined using the method of Mandel and Marmur (1968).

\section{Determination of dry weight of bioactive endophytic marine Streptomyces species}

The cells were separated from the culture broth by centrifugation at $4000 \mathrm{rpm}$ for $20 \mathrm{~min}$, washed twice with distilled water, dried at $60^{\circ} \mathrm{C}$ until reaching a constant weight, and then determined as gram biomass per liter of culture broth $\left(\mathrm{gl}^{-1}\right)$.

\section{Improvement of anti-MRSA metabolites productivity}

The optimization of the production of anti-MRSA metabolites was achieved in $250 \mathrm{ml}$ Erlenmeyer flasks containing $50 \mathrm{ml}$ of inorganic salts starch medium (ISP4) supplemented with $\mathrm{NaNO}_{3}$ as its nitrogen source, in $100 \%$ seawater. It was inoculated with Streptomyces sp. RS-9 and Streptomyces sp. MS-26 strains individually at a concentration of $10^{6} / \mathrm{ml}$ and incubated on a rotary shaker at $28^{\circ} \mathrm{C}$ and $180 \mathrm{rpm}$.

\section{Typical time course of antibiotic production by $R S-9$ and MS-26 strains}

The growth of each strain was determined periodically during 10 days of fermentation as $\mathrm{gl}^{-1}$ and the production of anti-MRSA metabolites was tested by measuring the diameters of inhibition zones.

\section{Effects of different temperature and $\mathrm{pH}$ on anti-MRSA production}

Both RS-9 and MS-26 strains were incubated at different temperatures $\left(25,27.5,30,35\right.$, and $\left.40^{\circ} \mathrm{C}\right)$ on a rotary shaker for 6 and 3 days, respectively, and then the optimum growth temperature for the highest antimicrobial activities against MRSA isolates by each strain was determined. The initial $\mathrm{pH}$ of the fermentation medium was tested $(4,5,6,7,8,9$, and 10$)$ at $27.5^{\circ} \mathrm{C}$ to de- 
termine the suitable initial $\mathrm{pH}$, which gave maximum antibiotic production.

\section{Effects of different carbon, nitrogen and metal ion sources on anti-MRSA production}

The impacts of following sources were evaluated: 1) different carbon sources, individually (D-glucose, D-galactose, lactose, maltose, sucrose, mannitol, cellulose, and glycerol, 1\%), instead of starch in medium; 2) inorganic nitrogen sources $\left(\mathrm{NH}_{4} \mathrm{NO}_{3},\left(\mathrm{NH}_{4}\right)_{2} \mathrm{SO}_{4}\right.$, and $\left.\mathrm{NH}_{4} \mathrm{H}_{2} \mathrm{PO}_{4}\right)$; 3) organic nitrogen sources (casein hydrolysate, peptone, yeast extract, tryptone, soybean, corn yellow extract, corn meal extract, peanut, fish extract, oat extract, and urea); 4) certain amino acids (glutamic acid, alanine, valine, arginine, tryptophan, asparagines, lysine, and methionine) at $0.2 \%$ final concentration (as N-base), and 5) metal ions $\left(\mathrm{MgSO}_{4}, \mathrm{FeSO}_{4}, \mathrm{MnCl}_{2}, \mathrm{ZnSO}_{4}, \mathrm{CuSO}_{4}\right.$, $\mathrm{NaCl}, \mathrm{CaCO}_{3}, \mathrm{~K}_{2} \mathrm{HPO}_{4}$ and $\mathrm{KH}_{2} \mathrm{PO}_{4}$ ) on anti-MRSA metabolites productivity on anti-MRSA production by both Streptomyces RS-9 and MS-26 strains.

\section{Gas Chromatography/Mass Spectrometry analysis $(G C / M S)$ of the volatile metabolites in Streptomyces sp. RS-9 and Streptomyces sp. MS-26 crude extracts}

The whole broth of each bioactive strain (Streptomyces sp. RS-9 and Streptomyces sp. MS-26) was extracted using ethyl acetate $(1: 1, \mathrm{v} / \mathrm{v})$. Each extract was concentrated and analyzed by GC/MS instrument at GC column with an inert gas (helium) oven temperature of $70^{\circ} \mathrm{C}$, and injector temperature of $200^{\circ} \mathrm{C}$ in spilt mode ratio 40 with a flow rate of $1.5 \mathrm{ml} / \mathrm{min}$. The MS ion source temperaturewas $200^{\circ} \mathrm{C}$, interface temperature was $240^{\circ} \mathrm{C}$, event time $0.5 \mathrm{~s}$, solvent cut time $5 \mathrm{~min}$ and MS start $5 \mathrm{~min}$, MS end time $35 \mathrm{~min}$. The mass spectra were recorded in electron ionization (EI) mode at $70 \mathrm{ev}$ as previously described by Sudha and Selvam (2012). Chromatogram integration and library searches were performed using the libraries of the Central Laboratory of Pesticides, Agricultural Research Center, Egypt.

\section{Extraction and isolation of anti-MRSA compounds from marine endophytic S. xanthochromogenus MS-26 strain}

The fermented broth of MS-26 strain (10 1) was collected and filtered by centrifugation at $10000 \mathrm{rpm}$ under cooling conditions $\left(4^{\circ} \mathrm{C}\right)$ for $10 \mathrm{~min}$, the $\mathrm{pH}$ of the clear supernatant was adjusted to 4.0 with $\mathrm{HCl}$, and then extracted with $2 \times$ EtOAc $(1: 1 \mathrm{v} / \mathrm{v})$. The mixture was shaken overnight and was subsequently kept under sta- tionary conditions for $60 \mathrm{~min}$ to separate the solvent from the aqueous phase. The extract was dried over anhydrous $\mathrm{Na}_{2} \mathrm{SO}_{4}$ and evaporated to dryness, producing yellow oil $(4.11 \mathrm{~g})$. The extract was defatted and the methanolic part $(2.98 \mathrm{~g}$ ) was pre separated by a column chromatography on silica gel (60-120 mesh) eluted with a linear gradient of $\mathrm{CHCl}_{3}$ and $\mathrm{MeOH}$. The active bactericidal fraction eluted with $\mathrm{CHCl}_{3} / \mathrm{MeOH}(95: 5)$ was further purified on Sephadex LH-20, eluted with methyl alcohol that finally gave $100.2 \mathrm{mg}$ of pure yellow powder (compound A), which finally constituted the major ingredient of a clear supernatant of $S$. xanthochromogenus MS-26 strains. On the other hand, after extracting the mycelium cake twice with methanol, the methanolic crude extract was extracted 3 times with ethyl acetate $(\mathrm{EtOAc})$. The EtOAc extract was fractionated by silica gel (60-120 mesh, $30.0 \mathrm{~g}$ ) column chromatography employing elution with a mixture of chloroform, methanol, and water $(90: 5: 5)$ to yield 3 fractions, one of them was active against MRSA isolates. The active fraction was further purified using Sephadex LH-20, eluted with $\mathrm{CHCl}_{3}$. As a result $63.11 \mathrm{mg}$ of a pure white powder compound (B) was obtained and described as shown below.

\section{Isolation of anti-MRSA compounds from marine endophytic Streptomyces sp. MS-26}

The fermented broth of MS-26 (101) was centrifuged at $10000 \mathrm{rpm}$ at $4^{\circ} \mathrm{C}$ for $10 \mathrm{~min}$ and then the cell-free supernatant was extracted twice with ethyl acetate (EtOAc) $(1: 1, \mathrm{v} / \mathrm{v}$ at $\mathrm{pH} 4.0)$. The mycelium cake was extracted twice with methanol. The thin layer chromatography (TLC) revealed that the extract of filtrate and mycelium did not contain the same constituents and had different $R f$ values. Therefore, they were not combined and were purified separately by a column chromatography on silica gel (60-120 mesh) and Sephadex LH-20. After each purification step, the antimicrobial activity of each fraction or pure compound was assayed against MRSA isolates using the disk diffusion method.

\section{Characterization of the anti-MRSA agents produced by endophytic Streptomyces sp. MS-26}

The characterization of compounds isolated from marine Streptomyces sp. MS-26 was performed using different methods. The functional groups were determined by Infrared spectra-Fourier transform 300 E Infrared (FT-IR) spectrometer using $\mathrm{KBr}$ disk, at the National 
Research Center, Egypt. The proton nuclear magnetic resonance $\left({ }^{1} \mathrm{H}\right.$ NMR) and mass spectra were determined at the Micro-analytical Center of Cairo University, Egypt.

\section{Determination of the MIC and MBC of pure compounds}

The MIC values of pure agents were determined against MRSA and CoNS isolates by a broth tube dilution procedure using two-fold dilution in $\mathrm{MH}$ broth at $37^{\circ} \mathrm{C}$ for $24 \mathrm{~h}$. MIC was determined as the lowest concentration of each compound showing no visible bacterial growth (Cappuccino and Sherman, 1999). MBC values were determined by sub-culturing $50 \mathrm{ml}$ from tubes that are not visibly turbid and spot inoculating onto $\mathrm{MH}$ plates. $\mathrm{MBC}$ values were determined as the lowest concentration of the tested compounds that prevented the growth of subcultures (Lavermicocca et al., 2003).

\section{Determination of anticancer activity (MTT assay)}

Cell viability was assessed as described by Mosmann (1983). Cells were suspended in Roswell Park Memorial Institute (RPMI) 1640 medium for human hepatocellular carcinoma (HepG2) and human colon cancer cells (HCT116), while Dulbecco's Modified Eagle's medium (DMEM) was used for human lung cancer cells (A549) with $1 \%$ antibiotic-antimycotic mixture $\left(10000 \mathrm{Uml}^{-1}\right.$ po-

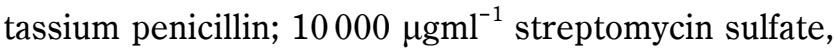

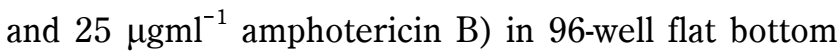
microplate at $37^{\circ} \mathrm{C}$, under $5 \% \mathrm{CO}_{2}$, using a water-jacketed carbon dioxide incubator (Sheldon, TC2323, Cornelius, OR, USA). After incubation, each culture medium was replaced with $100 \mathrm{ml}$ serum-free medium containing different concentrations of each compound, individually, for $48 \mathrm{~h}$ and then the media were aspirated and $40 \mu \mathrm{l}$ MTT salt $(2.5 \mu \mathrm{g} / \mathrm{ml})$ was added to each well and incubated for $4 \mathrm{~h}$ at $37^{\circ} \mathrm{C}$ under the same conditions. To stop the reaction and dissolve the formed crystals, $200 \mu \mathrm{l}$ of $10 \%$ sodium dodecyl sulfate (SDS) in deionized water was added to each well and incubated overnight at $37^{\circ} \mathrm{C}$. The absorbance was measured at $595 \mathrm{~nm}$ using a microplate multiwell reader (Bio-Rad Laboratories Inc. model 3350, Hercules, California, USA). A statistical significance was tested between the samples and the negative controls (cells with vehicle) using an independent t-test by SPSS11 program. $\mathrm{IC}_{50}$ values were determined by calculating the percentage of viability using the following formula: $\%$ of viability $=($ Mean test OD/Mean OD of the controls $) \times 100$.

\section{Results and discussion}

\section{Prevalence, isolation, and identification of clinical Staphylococcus isolates}

In this study, 41 cultures of Gram-positive Staphylococci were collected from 41 patients (22 males and 19 females) with age ranging from 1 to over 70 , from 2 different hospitals (Table 1). Among them, $73.2 \%$ were collected from El-Demerdash hospital and 26.8\% from Ain Shams Specialized Hospitals, which reflected a more frequent distribution of Staphylococci in general hospitals than in specialized ones. Moreover, the highest number of Staphylococci cultures (41.5\%) was collected from the people aged between 20 to 50 years (Table 1 ).

Table 1. Demographic and clinical data of collected cultures

\begin{tabular}{l|c|c}
\hline $\begin{array}{c}\text { Demographic } \\
\text { and clinical conditions }\end{array}$ & $\begin{array}{c}\text { Number } \\
\text { of isolates }\end{array}$ & $\begin{array}{c}\text { Percent } \\
\text { from total isolates } \\
{[\%]}\end{array}$ \\
\hline $\begin{array}{l}\text { Hospital } \\
\text { El-Demerdash }\end{array}$ & 30 & 73.2 \\
ain shams specialized & 11 & 26.8 \\
\hline Sex & & \\
male & 19 & 53.7 \\
female & & 46.3 \\
\hline Age & 1 & \\
$1-6$ & 2 & 2.4 \\
$6-12$ & 17 & 4.9 \\
$12-20$ & 12 & 4.9 \\
$20-50$ & 5 & 41.5 \\
$50-60$ & 2 & 29.3 \\
$60-70$ & & 12.2 \\
$>70$ & & 4.9 \\
\hline
\end{tabular}

On the other hand, as shown in Table 2, 29 specimens were collected from inpatients admitted to different hospitals and 9 different units $(10,4,4,4,2,2,1,1$, and 1 number of specimens were collected from the intensive care unit (ICU), medical medicine, surgery, orthopedic, ear nose and throat (ENT), neurology, emergency, hematology, and skin and venereal disease departments, respectively), while 12 (29.27) specimens were collected from outpatients. Thus patients from the ICU department represented $24.39 \%$ of all Staphylococci positive patients and $34.5 \%$ of all hospital inpatients, which indicated that hospitalization in the ICU has a significant risk factor for MRSA colonization and infection. These data are similar to those obtained by Sharaf and co- 
Table 2. Distribution of Staphylococci isolates in different hospital departments

\begin{tabular}{l|c|c}
\hline \multicolumn{1}{c|}{ Department } & $\begin{array}{c}\text { Number } \\
\text { of Staphylococci isolates }\end{array}$ & $\begin{array}{c}\text { Percent } \\
\text { from Staphylococci isolates }\end{array}$ \\
\hline I.C.U. & 10 & 24.39 \\
Emergency & 1 & 2.44 \\
Medical medicine & 4 & 9.76 \\
Surgery & 4 & 9.76 \\
E.N.T. & 2 & 4.88 \\
Neurology & 2 & 4.88 \\
Orthopedic & 4 & 9.76 \\
Hematology & 1 & 2.44 \\
Skin and Venereal Disease & 1 & 2.44 \\
Outpatient & 12 & 29.27 \\
\hline
\end{tabular}

Table 3. Morphological and biochemical characteristics of Staphylococcus aureus isolates

\begin{tabular}{l|c}
\hline \multicolumn{1}{c|}{ Physiological and biochemical test } & Characteristics \\
\hline $\begin{array}{l}\text { Morphological features } \\
\text { cell shape }\end{array}$ & cocci \\
gram stain & + \\
\hline Biochemical characteristics & + \\
catalase & + \\
slide coagulase test & + \\
tube coagulase test & + \\
DNase test & + \\
gelatin liquefaction test & + \\
acid production from mannitol fermentation & + \\
acid production from glucose fermentation & + \\
\hline Growth on selective media & + \\
Staphylococcus medium 110 & + (black) \\
baird parker & + \\
mannitol salt agar & + (gray black) \\
hemolysis on blood agar & + (blue) \\
Vogel Johnson agar & \\
oxacillin resistant screen agar base &
\end{tabular}

Table 4. Prevalence and distribution of Staphylococci and MRSA isolates in different clinical samples

\begin{tabular}{l|c|c|c|c}
\hline \multirow{2}{*}{ Specimen } & \multicolumn{2}{|c|}{ Staphylococci isolates } & \multicolumn{2}{c}{ MRSA isolates } \\
\cline { 2 - 5 } & number & percent [\%] & number & percent [\%] \\
\hline Wound (pus) & 15 & 36.6 & 9 & 50 \\
Sputum & 12 & 29.3 & 8 & 44.4 \\
Blood & 3 & 7.3 & 0 & 0 \\
Urine & 3 & 7.3 & 0 & 0 \\
Others & 8 & 19.5 & 1 & 5.6 \\
\hline
\end{tabular}

workers (2006) from the medical ICU of the Ain-Shams University Hospital and Sadaka and coworkers (2009) in ICUs of Alexandria Main University Hospital where they found that $35 \%$ and $43 \%$ of prevalence of $S$. aureus, respectively, compared to $28 \%$ detected by Awadalla and coworkers (2010) in Chest ICU patients. 
Table 5. Antibiotic sensitivity profile of MRSA isolates

\begin{tabular}{|c|c|c|c|c|}
\hline Class of antibiotics & $\begin{array}{c}\text { Number } \\
\text { of resistant MRSA }\end{array}$ & $\begin{array}{c}\text { Percent } \\
\text { of resistant MRSA } \\
{[\%]}\end{array}$ & $\begin{array}{c}\text { Number } \\
\text { of sensitive MRSA }\end{array}$ & $\begin{array}{c}\text { Percent } \\
\text { of sensitive MRSA } \\
{[\%]}\end{array}$ \\
\hline $\begin{array}{l}\text { Vancomycin } \\
\text { Cefoxitin } \\
\text { Cefotaxime } \\
\text { Cefazoline } \\
\text { Doxycycline } \\
\text { Oxytetracycline } \\
\text { Amikacin } \\
\text { Ciprofloxacin } \\
\text { Ofloxacin } \\
\text { Tienam } \\
\text { Erythromycin } \\
\text { Chloramphenicol } \\
\text { Nitrofurantoin } \\
\text { Sulfamethazole/Trimethoprim }\end{array}$ & $\begin{array}{l}- \\
18 \\
18 \\
17 \\
13 \\
16 \\
14 \\
17 \\
15 \\
18 \\
15 \\
16 \\
17 \\
14\end{array}$ & $\begin{array}{c}- \\
100 \\
100 \\
94.4 \\
72.2 \\
88.9 \\
77.8 \\
94.4 \\
83.3 \\
100 \\
83.3 \\
88.9 \\
94.4 \\
72.2\end{array}$ & $\begin{array}{l}18 \\
- \\
- \\
1 \\
5 \\
2 \\
4 \\
1 \\
3 \\
- \\
3 \\
2 \\
1 \\
4\end{array}$ & $\begin{array}{c}100 \\
- \\
- \\
5.6 \\
27.8 \\
11.1 \\
22.2 \\
5.6 \\
16.7 \\
- \\
16.7 \\
11.1 \\
5.6 \\
22.2\end{array}$ \\
\hline
\end{tabular}

Out of the 41 Staphylococcus cultures, 18 isolates (43.90\%) were identified as $S$. aureus according to their morphological, physiological, and biochemical features. These isolates were Gram positive cocci that appeared round in clusters and tetrads when viewed through a microscope. They showed positive results for the growth on the following media: mannitol salt agar (MSA, yellow growth), Staphylococcus medium 110, Baird parker agar base (black growth), Vogel and Johnson agar (gray black growth) and oxacillin resistance screening agar base (ORSAB, blue growth). They also showed positive results for the following tests: catalase test, coagulase test, DNase test, gelatin liquefaction test, hemolysis test at $37^{\circ} \mathrm{C}$ for $18-24 \mathrm{~h}$ on blood agar, and acid production from mannitol fermentation (Table 3 ). Since single phenotypic tests are ineffective for the identification of $S$. aureus, Najjuka and coworkers (2010) recommended a combination of various tests like the growth on MSA, DNase test, and tube coagulase test for the best identification of $S$. aureus. Procop and coworkers (2002) reported that besides the positive catalase test growth identification on MSA and DNase agar are the most reliable tests with $100 \%$ sensitivity and specificity for $S$. aureus. Gram staining, hemolysis on blood agar and coagulase tests had $69,77.5$, and $100 \%$ sensitivity with specificity equal to $44.64,64.29$ and $94.92 \%$. As reported by Cherkaoui and coworkers (2007), MRSA strains are characterized by the blue color of colonies when grown on the ORSAB medium. The highest percentage of Staphylococcus isolates and MRSA isolates were collected from wounds ( 36.6 and $50 \%$, respectively) followed by sputum (29.3 and $44.4 \%$, respectively) (Table 4). Our data, dealing with the prevalence of MRSA strains, are higher than those mentioned by Udo and coworkers (2008), Zaki and coworkers (1989), and Sadaka and coworkers (2009) (MRSA constituted of 32, 32, and $71 \%$ of the total $S$. aureus isolates obtained from Kuwait hospital and Alexandria main teaching hospital of Egypt in 2008, 1989, and 2009, respectively). Subsequently, an increase in the resistance to methicillin among the $S$. aureus isolates increased from $32 \%$ in 1989 to $71 \%$ in 2009 and to $100 \%$ in this study, which, on the one hand, reflects the emergence of multidrug resistant isolates in hospitals, and a dramatically increased incidence of hyper virulent community-associated MRSA in Egyptian hospitals, on the other hand. Similar to our results, Baddour and coworkers (2006) and Sadaka and coworkers (2009) reported that MRSA isolates that came from wounds represented 39.7 and $34 \%$ of total cultures, respectively.

\section{Susceptibility of MRSA isolates to different classes of antibiotics}

In Table 5, the resistance profiles of MRSA that exhibit resistance to vancomycin and other classes of antibiotics are presented. All MRSA isolates showed coresistance to many classes of antibiotics and thus were qualified as multidrug-resistant MRSA strains (MDR-MRSA). All tested isolates were sensitive to vancomycin. Whereas, the highest resistance among all MRSA isolates 
Table 6. Antagonistic activity of endophytic Actinomycetes against MRSA and MRCoNS isolates derived from El-Demerdash hospital

\begin{tabular}{|c|c|c|c|c|c|c|c|c|c|c|c|c|c|c|c|}
\hline \multirow{3}{*}{ Endophytes } & \multirow{3}{*}{ Isolation source } & \multicolumn{14}{|c|}{ Antagonistic activity against the clinical strains as diameter of inhibition zone } \\
\hline & & \multicolumn{13}{|c|}{ MRSA isolates } & \multirow{2}{*}{$\frac{\text { MRCoNS isolate }}{\text { D5 }}$} \\
\hline & & D1 & $\mathrm{D} 2$ & D3 & $\mathrm{D} 4$ & D6 & D7 & D8 & D9 & D10 & D11 & D12 & D13 & D14 & \\
\hline RS-1 & Sinularia sp. & +++ & + & - & + & +++ & +++ & + & +++ & - & +++ & + & & +++ & +++ \\
\hline RS-2 & Sarcophyton sp. & +++ & + & - & +++ & +++ & +++ & + & +++ & +++ & +++ & + & +++ & +++ & +++ \\
\hline RS-3 & Rhytisma sp. & +++ & - & ++ & +++ & - & +++ & + & +++ & +++ & +++ & + & +++ & +++ & +++ \\
\hline RS-4 & Sarcophyton sp. & +++ & - & ++ & +++ & ++ & +++ & + & +++ & +++ & +++ & + & +++ & +++ & +++ \\
\hline RS-5 & Sinularia sp. & +++ & - & ++ & +++ & - & +++ & + & +++ & +++ & - & + & + & +++ & \\
\hline RS-6 & Rhytisma sp. & +++ & +++ & +++ & +++ & +++ & +++ & +++ & +++ & ++ & +++ & +++ & +++ & +++ & +++ \\
\hline RS-7 & Sarcophyton sp. & +++ & +++ & +++ & +++ & - & +++ & +++ & +++ & - & +++ & +++ & +++ & +++ & +++ \\
\hline RS-8 & Rhytisma sp. & +++ & +++ & +++ & +++ & +++ & +++ & +++ & +++ & ++ & +++ & +++ & +++ & +++ & +++ \\
\hline RS-9 & Sinularia sp. & +++ & +++ & +++ & +++ & +++ & +++ & +++ & +++ & ++ & +++ & +++ & +++ & +++ & +++ \\
\hline RS-10 & Rhytisma sp. & + & + & - & + & +++ & +++ & +++ & - & + & - & + & + & +++ & +++ \\
\hline RS-11 & Sarcophyton sp. & +++ & - & - & + & - & + & +++ & - & - & +++ & + & + & +++ & ++ \\
\hline RS-12 & Rhytisma sp. & - & + & - & - & - & + & - & - & - & - & ++ & - & ++ & - \\
\hline RS-13 & Sinularia sp. & + & + & +++ & - & - & + & ++ & + & - & + & ++ & ++ & + & + \\
\hline RS-14 & Rhytisma sp. & + & +++ & & ++ & - & +++ & ++ & + & - & + & ++ & - & +++ & - \\
\hline RS-15 & Sarcophyton sp. & +++ & +++ & +++ & ++ & - & + & ++ & +++ & - & + & ++ & - & ++ & +++ \\
\hline RS-16 & Rhytisma sp. & - & +++ & - & +++ & - & + & ++ & + & - & + & ++ & & + & + \\
\hline RS-17 & Sarcophyton sp. & - & + & + & +++ & - & ++ & - & - & - & + & +++ & ++ & + & - \\
\hline RS-18 & Rhytisma sp. & - & - & - & - & - & ++ & ++ & +++ & + & - & - & +++ & +++ & - \\
\hline RS-19 & Sinularia sp. & + & + & + & +++ & + & ++ & - & - & - & +++ & +++ & - & + & +++ \\
\hline RS-20 & Sarcophyton sp. & + & + & + & + & + & + & ++ & ++ & +++ & +++ & + & + & ++ & + \\
\hline RS-21 & Sinularia sp. & - & - & - & - & - & - & - & - & +++ & - & - & - & - & - \\
\hline RS-22 & Sinularia sp. & +++ & +++ & - & - & + & + & +++ & +++ & ++ & - & +++ & - & - & + \\
\hline RS-23 & Sarcophyton sp. & - & - & +++ & + & + & & - & +++ & - & - & +++ & +++ & - & +++ \\
\hline RS-24 & Sinularia sp. & +++ & - & + & ++ & - & +++ & - & +++ & - & - & +++ & - & - & +++ \\
\hline RS-25 & Sarcophyton sp. & +++ & + & ++ & + & - & +++ & +++ & + & ++ & +++ & - & +++ & - & - \\
\hline MS-26 & Jellyfish & +++ & +++ & +++ & +++ & +++ & ++ & +++ & +++ & +++ & +++ & +++ & +++ & - & +++ \\
\hline
\end{tabular}

+++ - highly active (16-20 mm), ++ - active (10-15 mm), + - weakly active (>10 mm), “-” - no activity, MRSA - methicillin-resistant Staphylococcus aureus, MRCoNS - methicillin-resistant coagulase-negative Staphylococci, RS - Red Sea, MS - Mediterranean Sea, D - refers to clinical isolates derived from El-Demerdash hospital 
Table 7. Antagonistic activity of endophytic Actinomycetes against MRSA and MRCoNS isolates derived from Ain Shams Specialized hospital

\begin{tabular}{|c|c|c|c|c|c|c|c|}
\hline \multirow{3}{*}{ Endophytes } & \multirow{3}{*}{ Isolation source } & \multicolumn{6}{|c|}{ Antagonistic activity against the clinical isolates as diameter of inhibition zone } \\
\hline & & \multicolumn{5}{|c|}{ MRSA isolates } & \multirow{2}{*}{$\frac{\text { MRCoNS isolate }}{\text { AS-15 }}$} \\
\hline & & AS-16 & AS-17 & AS-18 & AS-19 & AS-20 & \\
\hline RS-1 & Sinularia sp. & +++ & & +++ & +++ & + & +++ \\
\hline RS-2 & Sarcophyton sp. & - & +++ & +++ & +++ & + & +++ \\
\hline RS-3 & Rhytisma sp. & - & +++ & +++ & +++ & - & +++ \\
\hline RS-4 & Sarcophyton sp. & - & - & +++ & +++ & + & +++ \\
\hline RS-5 & Sinularia sp. & - & - & +++ & - & + & +++ \\
\hline RS-6 & Rhytisma sp. & ++ & +++ & +++ & +++ & + & +++ \\
\hline RS-7 & Sarcophyton sp. & - & +++ & +++ & - & + & +++ \\
\hline RS-8 & Rhytisma sp. & ++ & +++ & +++ & +++ & + & +++ \\
\hline RS-9 & Sinularia sp. & +++ & +++ & +++ & +++ & + & +++ \\
\hline RS-10 & Rhytisma sp. & - & +++ & +++ & - & + & - \\
\hline RS-11 & Sarcophyton sp. & - & - & +++ & +++ & + & - \\
\hline RS-12 & Rhytisma sp. & - & + & - & +++ & + & - \\
\hline RS-13 & Sinularia sp. & ++ & + & - & + & ++ & + \\
\hline RS-14 & Rhytisma sp. & - & + & - & - & +++ & + \\
\hline RS-15 & Sarcophyton sp. & - & + & - & - & + & +++ \\
\hline RS-16 & Rhytisma sp. & - & - & - & +++ & + & + \\
\hline RS-17 & Sarcophyton sp. & - & + & - & - & +++ & - \\
\hline RS-18 & Rhytisma sp. & - & - & - & +++ & - & - \\
\hline RS-19 & Sinularia sp. & +++ & ++ & - & - & - & ++ \\
\hline RS-20 & Sarcophyton sp. & ++ & ++ & +++ & +++ & +++ & ++ \\
\hline RS-21 & Sinularia sp. & - & - & - & - & - & - \\
\hline RS-22 & Sinularia sp. & - & +++ & +++ & +++ & - & +++ \\
\hline RS-23 & Sarcophyton sp. & - & +++ & +++ & - & - & +++ \\
\hline RS-24 & Sinularia sp. & - & + & ++ & ++ & +++ & +++ \\
\hline RS-25 & Sarcophyton sp. & + & ++ & + & + & +++ & + \\
\hline MS-26 & Jellyfish & +++ & +++ & + & +++ & +++ & ++ \\
\hline
\end{tabular}

+++ - highly active $(16-20 \mathrm{~mm}),++-$ active $(10-15 \mathrm{~mm}),+-$ weakly active $(>10 \mathrm{~mm})$, MRSA - methicillin resistant Staphylococcus aureus, MRCoNS - methicillin resistant coagulase negative Staphylococci, RS - Red Sea, MS - Mediterranean Sea, AS - refers to clinical isolates derived from Ain Shams Specialized hospital

(100\%) was reported for cefoxitin, cefotaxime, and tienam; followed by nitrofurantoin, ciprofloxacin, and cefazoline ( $94.4 \%$ for each). The highest susceptibility (27.8\%) was detected for doxycycline and sulfamethoxazole/trimethoprim followed by amikacin (22.2\%). The multidrug resistant phenotype is a characteristic feature of the MRSA strains and the antibiotic resistance is common for many classes of antibiotics such as aminoglycosides, macrolides, and fluoroquinolones. Nevertheless, all strains were sensitive to vancomycin (Awadalla et al., 2010; Kannan, 2011).

\section{Isolation of endophytic marine Actinomycetes}

In the current study, 25 endophytic Actinomycetes isolates were obtained from soft corals Sinularia sp.; Sarcophyton sp. and Rhytisma sp., which were collected from Hurghada of the Red Sea. One Actinomycete strain (MS-26) was isolated from some unidentified jellyfish collected from Sidi Bishr shore, Alexandria, the Mediterranean Sea (Table 6). In agreement with our study, Nithyanand and Pandian (2009) and El-Bondkly and coworkers (2012b) had reported that marine soft corals are novel, rich sources of actinobacteria possessing po- 
Table 8. Cultural and morphological properties of the selected Actinomycete isolates RS-9 and MS-26

\begin{tabular}{|c|c|c|c|c|c|c|c|c|}
\hline \multirow{3}{*}{ Medium } & \multicolumn{8}{|c|}{ Actinomycete isolates } \\
\hline & \multicolumn{4}{|c|}{ RS-9 } & \multicolumn{4}{|c|}{ MS-26 } \\
\hline & Growth & Aerial mycelium & Substrate mycelium & $\begin{array}{c}\text { Soluble } \\
\text { pigment }\end{array}$ & Growth & Aerial mycelium & Substrate mycelium & $\begin{array}{l}\text { Soluble } \\
\text { pigment }\end{array}$ \\
\hline Sucrose nitrate agar & good & white & yellowish white & none & scanty & pale gray & whitish yellow & none \\
\hline Glucose nitrate agar & good & white & yellowish white & none & excellent & gray & yellow & none \\
\hline Glucose asparagine agar & good & white & yellowish white & none & good & gray & yellow & none \\
\hline Inorganic salt asparagine & good & white & yellowish white & none & good & dark gray & yellow & none \\
\hline Nutrient agar & moderate & white & yellowish white & none & poor & pale gray & pale yellow & none \\
\hline Glucose peptone agar & good & white & yellowish white & none & excellent & dark gray & light yellow & none \\
\hline Starch nitrate agar & good & white & yellowish white & none & excellent & dark gray & yellowish orange & none \\
\hline Czapek's agar & good & white & yellowish white & none & moderate & gray & pale orange & none \\
\hline ISP1 & good & white & yellowish white & none & excellent & dark gray & white & none \\
\hline ISP2 & good & white & yellowish white & none & excellent & dark gray & whitish yellow & none \\
\hline ISP3 & moderate & white & yellowish white & none & poor & pink gray & yellow & none \\
\hline ISP4 & good & white & yellowish white & none & good & yellowish gray & yellow & none \\
\hline ISP5 & good & white & yellowish white & none & excellent & dark gray & deep yellow & none \\
\hline ISP6 & good & white & yellowish white & none & excellent & dark gray & yellow & none \\
\hline ISP7 & moderate & white & yellowish white & pale yellow & moderate & whitish gray & pale yellow & none \\
\hline
\end{tabular}


Table 9. Physiological and biochemical characteristics of RS-9 and MS-26 isolates

\begin{tabular}{|c|c|c|c|c|c|}
\hline \multirow{2}{*}{$\begin{array}{c}\text { Physiological } \\
\text { and biochemical test }\end{array}$} & \multicolumn{2}{|c|}{ Characteristics } & \multirow{2}{*}{ Physiological and biochemical test } & \multicolumn{2}{|c|}{ Characteristics } \\
\hline & RS-9 & MS-26 & & RS-9 & MS-26 \\
\hline Formation of melanoid pigment & - & + & $\mathrm{G}+\mathrm{C}(\mathrm{Mol} \%)$ & $69 \%$ & $70.5 \%$ \\
\hline $\mathrm{H}_{2} \mathrm{~S}$ production & - & + & Cell wall amino acids (type I) & LL-DAP, Gly & LL-DAP, alan, glyc, gluta \\
\hline Reduction of $\mathrm{NaNO}_{3}$ & + & + & Whole-cell sugars & Gala, gluc, xylose & Ribo, xylo, arab, gala, gluc \\
\hline Liquefaction of gelatin & + & + & Major fatty acids (\%) & & \\
\hline Milk coagulation & + & + & ai-C13: 0 & 0.42 & 0 \\
\hline Milk peptonization & + & + & i-C13: 0 & 0.56 & 0 \\
\hline Utilization of (1\%) & & & ai-C14: 0 & 0 & 0.94 \\
\hline L-Arabinose & - & - & C14: 0 & 0 & 0.54 \\
\hline L-Xylose & - & - & ai-C15: 0 & 11.00 & 30.84 \\
\hline D-Glucose & + & + & i-C15: 0 & 6.35 & 3.20 \\
\hline D-Fructose & + & + & C15: 0 & 0 & 0.50 \\
\hline D-Galactose & + & + & iso-C15:0 & 11.25 & 1.00 \\
\hline L-Rhamnose & + & - & anteiso-C15:0 & 10.28 & 11.25 \\
\hline Sucrose & + & + & i-C16:0 & $25 \cdot 50$ & 18.40 \\
\hline 1- Inositol & - & + & C16: 0 & 1.14 & 9.14 \\
\hline Raffinose & - & - & iso-C16: 0 & 25.15 & 0 \\
\hline D-Mannitol & + & + & ai-C17: 0 & 0 & 13.4 \\
\hline Glycerol & + & + & iso-C17: 0 & 0 & 1.32 \\
\hline D-Mannose & + & + & anteiso-C17: 0 & 0 & 8.00 \\
\hline D-Ribose & - & + & $\mathrm{i}-\mathrm{C} 17: 0$ & 0 & 1.47 \\
\hline Lactose & + & + & C17: 0 & 4.60 & 0 \\
\hline Maltose & + & + & C18: $1 v 8 c$ & 2.75 & 0 \\
\hline Utilization of & & & C18: 0 & 1.00 & 0 \\
\hline Casein & + & + & Major quinones (\%) & & \\
\hline L-Valine & + & + & MK-8(H8) & 0 & 2.50 \\
\hline L-Phenylalanine & - & + & MK-9(H4) & 3.00 & 40.12 \\
\hline L-Histidine & + & + & MK-9(H6) & 10.09 & 11.00 \\
\hline L-Cysteine & - & - & MK-9(H8) & 51.31 & 15.17 \\
\hline L-Hydroxyproline & - & - & MK-9(H10) & 1.3 & 1.42 \\
\hline Hydrolysis of & & & MK-10(H0) & 0 & 25.13 \\
\hline Starch & + & + & MK-10(H6) & 17.14 & 0 \\
\hline Cellulose & + & + & MK-10(H8) & 8.74 & 4.66 \\
\hline Chitin & + & + & MK-10(H4) & 6.31 & 0 \\
\hline Citrate & + & + & MK-10(H10) & 2.11 & 0 \\
\hline Oxalate & + & - & Characteristic phospholipids & & \\
\hline Temperature range $\left({ }^{\circ} \mathrm{C}\right)$ & $10-40$ & $15-40$ & Phosphatidylethanolamine (PE) & + & + \\
\hline $\mathrm{pH}$ range & $5-10$ & $5-10$ & Phosphatidylglycerol (PG) & + & - \\
\hline Growth at $45^{\circ} \mathrm{C}$ & - & - & Diphosphatidylglycerol (DPG) & + & + \\
\hline $\mathrm{NaCl}$ tolerance & $13 \%$ & $18 \%$ & Phosphatidylinositol(PI) & + & - \\
\hline Sodium azide $(0.1 \%)$ & - & + & Phosphatidylcholine (PC) & + & - \\
\hline Phenol (0.1\%) & + & - & Phosphatidylinositol mannosides (PIM) & - & + \\
\hline
\end{tabular}

Alan - alanine, glyc - glycine, gluta - glutamine, gala - galactose, gluc - glucose, ribo - ribose, xylo - xylose, arab - arabinose 


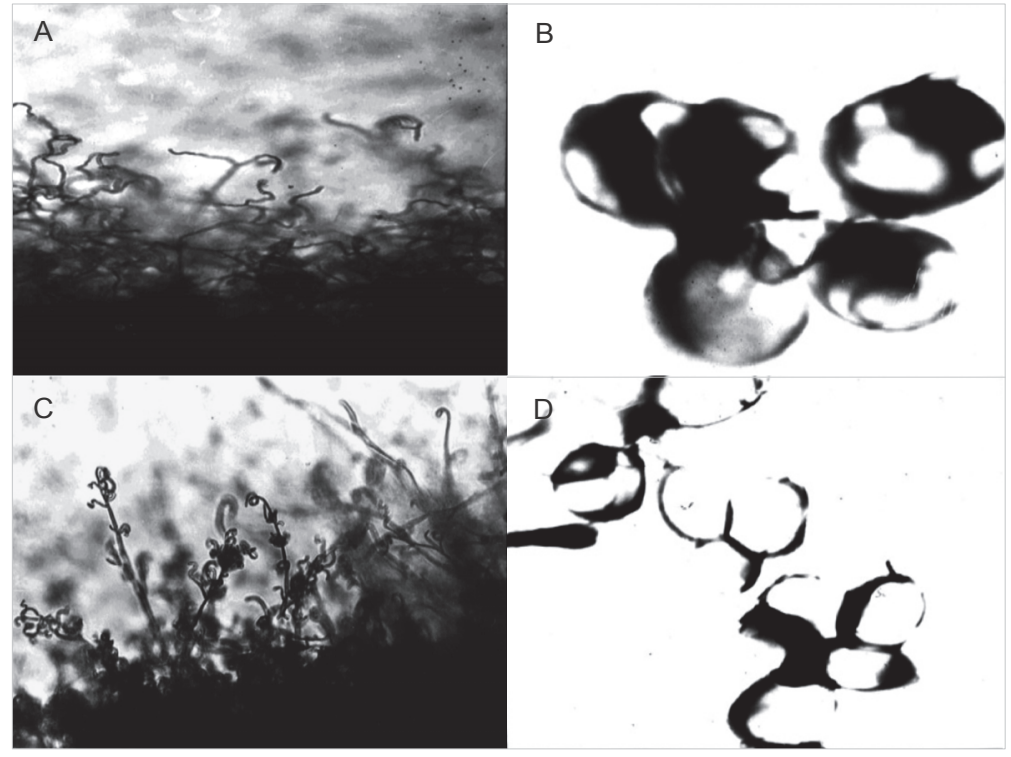

Fig. 1. Microphotograph of spore chains morphology and a transmission electron micrograph of the spore morphology of marine endophytic Streptomyces sp. RS-9 (A and B) and Streptomyces sp. MS-26 (C and D) isolates

tentially active natural compounds. Moreover, El-Bondkly and coworkers (2012a) showed that the dominant genera isolated from different soft corals from the Red Sea were Streptomyces, Nocardioposis, and Pseudonocardia. Streptomyces strains 1996 and 7409 from jellyfish, obtained from El-Agami coast of the Mediterranean Sea, have been described as sources of different bioactive compounds by El-Gendy and coworkers (2008a).

\section{Inhibitory activity of endophytic marine Actinomycetes against MRSA isolates}

Screening of Actinomycetes isolates (26 isolates) for their inhibitory activity against 18 isolates of MDRMRSA and 2 isolates of CoNS-D5 and CoNS-AS15 revealed that all Actinomycetes isolates showed varied inhibitory activity toward all tested clinical isolates (Table 6 and Table 7). Among them, RS9 and MS-26 isolates were selected for further studies, because they represented 2 endophytic Actinomycetes obtained from different marine invertebrates (the soft coral Sarcophyton sp. and jellyfish), living in two different marine ecosystems (the Red and Mediterranean Seas, respectively). Similarly, Valli and coworkers (2012) indicated marine Streptomyces species as a fruitful source for anti-Staphylococcus agents. For instance, Streptomyces aburaviensis Kut- 8 produced active antibiotics against $S$. aureus, and other Gram positive and Gram negative bacteria (Thumar et al., 2010).

\section{Morphological and biochemical properties of selected anti-MRSA isolates}

The characterization of RS-9 and MS-26 isolates was performed using a polyphasic approach. Both strains possessed a range of phenotypic and chemotaxonomic characteristics (Fig. 1 as well as Table 8 and Table 9) typical for members of the genus Streptomyces, according to Zhou and coworkers (1998), Kawato and Shinobu (1959), Szabo and coworkers (1975), Williams and coworkers (1989), Kämpfer and coworkers (1991), Shirling and Gottlieb (1966), Hasegawa and coworkers (1983), Lechevalier and coworkers (1977), Minnikin and coworkers (1977), Butte (1983) and Mandel and Marmur (1968).

\section{Identification of $\mathrm{RS}-9$ isolate}

RS-9 isolate is an aerobic Gram-positive Actinomycete, which forms an extensively branched substrate mycelium and aerial hyphae that differentiate into spiral spore chains with smooth surfaces (Fig. 1A and Fig. 1B). As shown in Table 8, a white aerial mycelium with yellowish white substrate mycelium was produced on all the growth media used. The results of tests for the formation of soluble pigments (with the exception of ISP7 medium), melanin formation, and $\mathrm{H}_{2} \mathrm{~S}$ production were negative, but tests for gelatin liquefaction, milk coagulation, milk peptonization, and nitrate reduction gave positive results (Table 8 and Table 9). Further cultural properties of the tested bacterial strains, on different 
agar media, are shown in Table 8 . Bacteria grew well at temperatures between $10^{\circ} \mathrm{C}$ and $40^{\circ} \mathrm{C}$ but not at $45^{\circ} \mathrm{C}$, and at $\mathrm{pH}$ values between 5.0 and 10.0. Growth was also observed in a growth medium supplemented with $0.1 \%$ sodium azide (Table 9 ). In addition, the RS-9 isolate degraded casein, starch, cellulose, chitin, citrate, and oxalate. Isolate RS-9 could utilize glucose, fructose, mannose, galactose, rhamnose, sucrose, lactose, maltose, mannitol, and glycerol as sole carbon source as well as L-valine and L-histidine as sole nitrogen source for energy and growth, but not arabinose, xylose, L-inositol, raffinose, ribose, L-phenylalanine, L-cysteine, and L-hydroxyproline (Table 9). The cell wall contained LL-diaminopimelic acid (LL-DAP) and glycine (cell wall type I) with whole-cell sugars of galactose, glucose, and xylose. Strain RS-9 exhibited fatty acid profile typical for Streptomyces (type IIc) comprising of ai-C13:0 (0.42\%), i-C13:0 (0.56\%), ai-C15:0 (11.0\%), i-C15:0 (6.35\%), iso-C15:0 (11.25\%), anteiso-C15:0(10.28\%), i-C16:0(25.5\%), C16:0 (1.14\%), iso-C16:0 (25.15\%), C17:0 (4.6\%), C18:1v8c (2.75\%), and C18:0 (1.0\%) (Table 9). The menaquinones includeMK-9(H4) (3.0\%), MK-9(H6) (10.09\%), MK-9(H8) (51.31\%), and MK-9(H10) (1.3\%), MK-10(H4) (6.31\%), MK-10(H6) (17.14\%), MK-10(H8) (8.74\%), MK-10(H10) (2.11\%) (Table 9$)$. The diagnostic phospholipid pattern was of type II containing, phosphatidylethanolamine (PE), phosphatidylglycerol (PG), diphosphatidylglycerol (DPG), phosphatidylinositol (PI), and phosphatidylcholine (PC). Moreover, the $\mathrm{G}+\mathrm{C}$ content of DNA was $69 \%$ (Table 9). The strain was proven to be Streptomyces vastus, and was given the tentative name Streptomyces vastus RS9.

\section{Identification of MS-26 isolate}

MS-26 forms an extensively branched substrate mycelium and aerial hyphae, which carries smooth-surfaced spores in hocked spore chains (Fig. 1C and Fig. 1D). The growth of a grayish aerial spore mass with a white to yellowish-orange substrate mycelium was observed (Table 8). Diffusible pigments were not formed, contrary to $\mathrm{H}_{2} \mathrm{~S}$ and melanin pigments (Table 8 and Table 9). Growth was observed between 15 and $40^{\circ} \mathrm{C}$ and pH 5.0 to 10.0 in the presence of $18 \%(\mathrm{w} / \mathrm{v}) \mathrm{NaCl}$ and $0.1 \%$ (w/v) sodium azide, but not when $0.1 \%$ phenol was added (Table 9). MS-26 isolate was able to perform liquefaction of gelatin and hydrolysis of starch, casein, cellulose, chitin, and citrate, but unable to utilize arabinose, xylose,
L-rhamnose, raffinose, L-cysteine, L-hydroxyproline, and oxalate (Table 9). Amino acids detected in the peptidoglycan layer of MS-26 strain were LL-diaminopimelic acid (LL-DAP), alanine, glycine, and glutamic acid (cell wall type I); the whole-cell hydrolysates contained ribose, $x y-$ lose, arabinose, galactose, and glucose (Table 9). The fatty acid profile was composed of ai-C14:0 (0.94\%), C14:0 (0.54\%), ai-C15:0 (30.84\%), i-C15:0 (3.2\%), C15:0 (0.50\%), iso-C15:0 (1.0\%), anteiso-C15:0 (11.25\%), i-C16 : 0 (18.4\%), C16:0 (9.14\%), ai-C17:0 (13.4\%), i-C17 : 0 (1.47\%), iso-C17:0 (1.32\%) and anteiso-C17:0 (8.0\%) (fatty acid type 2c sensu) (Table 9$)$. The predominant menaquinones were MK-8(H8) (2.5\%), MK-9(H4) (40.12\%), MK-9(H6) (11.0\%), MK-9(H8) (15.17\%), MK9(H10) (1.42\%), MK-10(H0) (25.13\%) and MK-10(H8) (4.66\%) while main polar lipids were phosphatidylethanolamine (PE), diphosphatidylglycerol (DPG), and phosphatidylinositol mannosides (PIM) (phospholipid type II sensu). The $\mathrm{G}+\mathrm{C}$ content of genomic DNA of MS-26 was $70.5 \%$ (Table 9). The strain was identified as Streptomyces xanthochromogenus, for which we suggested the name Streptomyces xanthochromogenus MS-26.

Optimization of culture conditions for the production of bioactive metabolites by $S$. vastus $R S-9$, and $S$. xanthochromogenus MS-26

\section{The time course of the production of anti-MRSA metabolites}

The data presented in Figure 2A denote that the incubation period controlled by the relationship between 2 phases of Streptomyces growth (trophophase and idiophase) showed a profound effect on the growth and antibiotic activity of both marine endophytic $S$. vastus RS-9 and $S$. xanthochromogenus MS-26 strains during 10 days of incubation. A complete growth was achieved after day 6 and day 7 for RS-9 and MS-26, respectively. The formation of antibiotic agents by both strains was initiated after the day 1 of incubation (the diameters of inhibition zones were 10 and $12 \mathrm{~mm}$, respectively) and it reached their maximum concentrations after the day 6 and day 3 of growth, with 29 and $27 \mathrm{~mm}$ diameters of inhibition zones, respectively (i.e., both trophophase and idiophase were associated) (Fig. 2A). An early production of antibiotics until trophophase in $S$. vastus RS-9 and $S$. xanthochromogenus MS-26 meant that they were relatively resistant to the antibiotics they produced. Moreover, the data indicated that the fermentation time of bioactive metabolites was shortened to 6 days for 


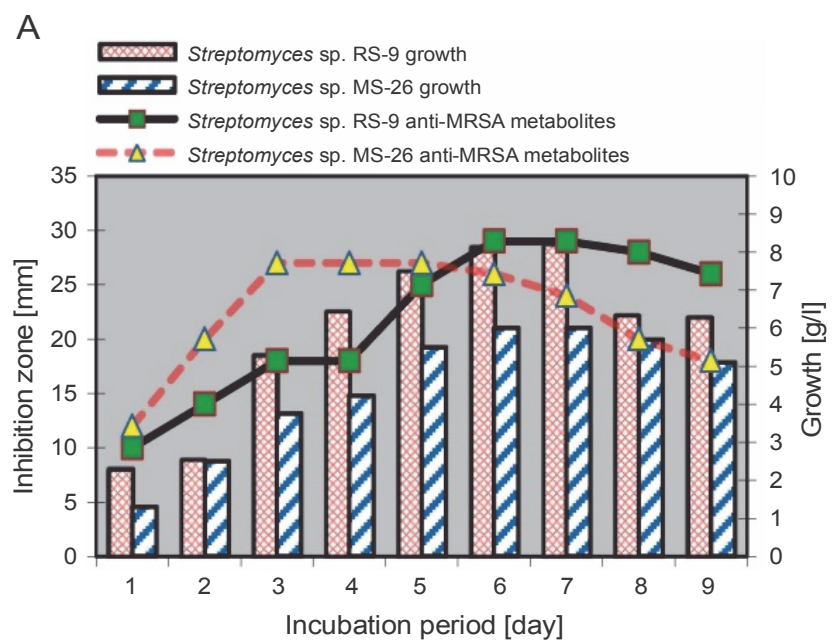

B
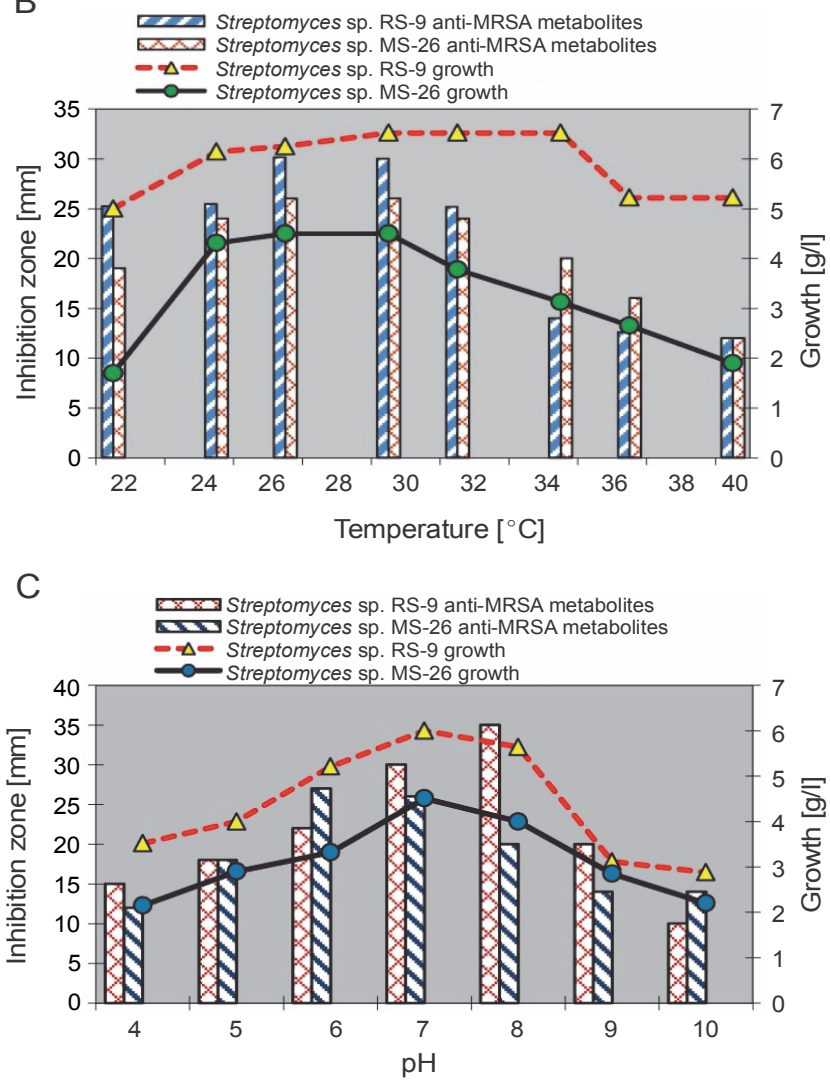

Fig. 2. A) Typical time course, B) effect of the incubation temperature and $\mathrm{C}$ ) initial $\mathrm{pH}$ on the growth and anti-MRSA metabolites production by endophytic Streptomyces species RS-9 and MS-26

S. vastus RS-9 and to 3 days for $S$. xanthochromogenus MS-26, which may give great advantages in industrial applications for increasing the production efficiency. Similar results were observed for the secondary metabolism in Actinomycetes. Atta and coworkers (2011) recorded a 3-day incubation period for the maximum antimicrobial activity by Streptomyces cyaneus AZ-13Zc, while El-Nagar and coworkers (2006) reported a 6-day incubation period for the maximum meroparamycin production by Streptomyces sp. MAR01.

\section{The effect of the incubation temperature on the production of anti-MRSA metabolites}

Temperature is a physical factor that plays a significant role in the growth and metabolism of microorganisms. The data given in Figure 2B indicate that the maximum production of the antimicrobial agent was obtained for $S$. vastus RS-9 and $S$. xanthochromogenus MS-26 at $27.5^{\circ} \mathrm{C}$ (the diameters of inhibition zones were 30.14 and 26.0, respectively) but the maximum growth was reached at $\left(6.52\right.$ and $\left.4.5 \mathrm{gl}^{-1}\right)$ at 30 and $27.5^{\circ} \mathrm{C}$, respectively. A higher or lower incubation temperature had an adverse effect on the growth and lowered the productivity of anti-MRSA metabolites. These data are in agreement with the productivity of meroparamycin from Streptomyces sp. MAR 01 were grown at $30^{\circ} \mathrm{C}$ by El-Naggar and coworkers (2006), but El-Gendy and coworkers (2008b) obtained different results for the maximum ayamycin production by marine Nocardia sp. ALAA-2000 at $35^{\circ} \mathrm{C}$.

\section{The effect of the initial pH of the medium on the production of anti-MRSA metabolites}

As illustrated in Figure 2C, the highest growth $\left(6.0 \mathrm{gl}^{-1}\right)$ and anti-MRSA activity yields (the diameter of the inhibition zone, $35.0 \mathrm{~mm}$ ) of $S$. vastus RS-9 were obtained at $\mathrm{pH} 7.0$ and 8.0, respectively. The highest growth $\left(4.51 \mathrm{gl}^{-1}\right)$ at $\mathrm{pH} 7.0$ and at the highest level of anti-MRSA substances (the diameter of the inhibition zone, $27 \mathrm{~mm}$ ) at $\mathrm{pH} 6.0$ were observed for $S$. xanthochromogenus MS-26. The $\mathrm{pH}$ value of the culture medium plays a significant role in the growth and metabolism by affecting the enzymatic reactions of different metabolic processes involved in cell permeability. This has been demonstrated by El-Gendy and coworkers (2008b) when the production of ayamycin by Nocardia sp. ALAA-2000 was successively improved with an increase in the $\mathrm{pH}$ to 7.5 .

\section{The effect of different carbon sources on the production of anti-MRSA metabolites}

Carbon compounds constitute a major requirement for the growth as they are being used in different metabolic processes, resulting in the production of primary and secondary metabolites (including the anti-MRSA metabolites). Glucose, followed by glycerol, gave the 
Table 10. Effect of different carbon sources on the production of anti-MRSA metabolites from $S$. vastus RS-9 and $S$. xanthochromogenus MS-26 strains

\begin{tabular}{l|c|c|c|c}
\hline \multirow{2}{*}{$\begin{array}{c}\text { Carbon } \\
\text { sources }\end{array}$} & $\begin{array}{c}\text { RS-9 } \\
\text { Dry weight } \\
{\left[\mathrm{gl}^{-1} \text { ] }\right.}\end{array}$ & $\begin{array}{c}\text { Anti-MRSA } \\
\text { (diameter of inhibition zone) } \\
{[\mathrm{mm}]}\end{array}$ & $\begin{array}{c}\text { MS-26 } \\
\text { Dry weight } \\
{\left[\mathrm{gl}^{-1}\right]}\end{array}$ & $\begin{array}{c}\text { Anti-MRSA } \\
\text { (diameter of inhibition zone) } \\
{[\mathrm{mm}]}\end{array}$ \\
\hline Control & 7.15 & 20.0 & 5.60 & 19.0 \\
Glucose & 8.71 & 30.0 & 5.32 & 11.0 \\
Galactose & 4.16 & 11.0 & 3.92 & 17.0 \\
lactose & 4.52 & 15.0 & 3.90 & 20.1 \\
Sucrose & 7.10 & 20.0 & 4.18 & 27.0 \\
Maltose & 9.22 & 18 & 4.00 & 31.12 \\
Glycerol & 9.14 & 25 & 5.08 & 31.5 \\
Mannitol & 7.00 & 22 & 3.91 & 15.7 \\
Cellulose & 5.00 & 15 & 3.55 & 18.9 \\
\hline
\end{tabular}

Table 11. Effect of different nitrogen sources on the production of anti-MRSA metabolites from $S$. vastus RS-9 and $S$. xanthochromogenus MS-26 strains

\begin{tabular}{|c|c|c|c|c|}
\hline \multirow[b]{2}{*}{ Nitrogen source } & \multicolumn{2}{|r|}{ RS-9 } & \multicolumn{2}{|r|}{ MS-26 } \\
\hline & $\begin{array}{l}\text { Dry } \\
\text { weight } \\
{\left[\mathrm{gl}^{-1}\right]}\end{array}$ & $\begin{array}{c}\text { Anti-MRSA } \\
\text { (diameter of inhibition zone) } \\
{[\mathrm{mm}]}\end{array}$ & $\begin{array}{c}\text { Dry } \\
\text { weight } \\
{\left[\mathrm{gl}^{-1}\right]}\end{array}$ & $\begin{array}{c}\text { Anti-MRSA } \\
\text { diameter of inhibition zone) } \\
{[\mathrm{mm}]}\end{array}$ \\
\hline $\mathrm{NaNO}_{3}$ (control) & 9.55 & 28.0 & 5.0 & 31.18 \\
\hline $\mathrm{NH}_{4} \mathrm{NO}_{3}$ & 9.67 & 20.19 & 5.0 & 32.14 \\
\hline$(\mathrm{NH} 4)_{2} \mathrm{SO}_{4}$ & 8.55 & 13.10 & 4.85 & 24.52 \\
\hline $\mathrm{NH} 4 \mathrm{H}_{2} \mathrm{PO}_{4}$ & 7.45 & 16.92 & 4.76 & 22.17 \\
\hline Casein & 7.96 & 25.49 & 4.79 & 20.91 \\
\hline Peptone & 8.42 & 38.14 & 5.62 & 25.19 \\
\hline Yeast extract & 7.18 & 38.35 & 6.04 & 27.00 \\
\hline Tryptone & 8.50 & 28.00 & 5.76 & 30.12 \\
\hline Soybean & 6.18 & 30.00 & 5.00 & 41.50 \\
\hline Corn yellow extract & 7.22 & 20.28 & 6.21 & 28.12 \\
\hline Corn meal extract & 10.0 & 30.52 & 5.88 & 28.00 \\
\hline Peanut & 8.00 & 39.00 & 6.00 & 32.90 \\
\hline Fish meal extract & 9.00 & 22.00 & 7.16 & 34.62 \\
\hline Oat extract & 4.99 & 18.00 & 2.25 & 12.52 \\
\hline Urea & 6.14 & 13.61 & 3.9 & 18.00 \\
\hline L-Glutamic acid & 4.00 & 17.80 & 3.00 & 22.00 \\
\hline L-Alanine & 3.16 & 14.52 & 2.65 & 11.70 \\
\hline L-Valine & 4.22 & 38.50 & 3.50 & 38.00 \\
\hline L-Arginine & 5.51 & 37.18 & 4.77 & 35.78 \\
\hline L-Tryptophan & 4.90 & 20.11 & 2.90 & 30.68 \\
\hline L-Asparagine & 5.70 & 35.00 & 4.91 & 40.50 \\
\hline L-Lysine & 6.7 & 30.18 & 3.78 & 18.75 \\
\hline Methionine & 4.57 & 26.50 & 5.00 & 42.20 \\
\hline Peanut + L-Valine & 7.48 & 44.00 & - & - \\
\hline L-Methionine+ L-Asparagine+ Soybean & - & - & 5.99 & 47.4 \\
\hline
\end{tabular}

highest productivity of anti-MRSA metabolites (the diameters of inhibition zones were 30 and $25 \mathrm{~mm}$, respectively) by $S$. vastus RS-9 strain, maltose supported the maximum growth yield $\left(9.22 \mathrm{gl}^{-1}\right)$ - Table 10 . Vasavada and coworkers (2006) and Kumar and coworkers (2011) indicated D-glucose as the best inducer for the antibiotic production by Streptomyces sannanensis and marine Amycolatopsis alba. On the other hand, glycerol, followed by maltose, were favored carbon sources for the maximum production of anti-MRSA metabolites in 
Table 12. Effect of metal ion elimination from the fermentation medium on the production of anti-MRSA metabolites from $S$. vastus RS-9 and $S$. xanthochromogenus MS-26 strains

\begin{tabular}{l|c|c|c|c}
\hline \multirow{2}{*}{\multicolumn{1}{c|}{ Excluded metal ion }} & \multicolumn{2}{|c|}{ RS-9 } & \multicolumn{2}{c}{ MS-26 } \\
\cline { 2 - 5 } & $\begin{array}{c}\text { Dry weight } \\
{\left[\mathrm{gl}^{-1}\right]}\end{array}$ & $\begin{array}{c}\text { Residual anti-MRSA activity } \\
{[\%]}\end{array}$ & $\begin{array}{c}\text { Dry weight } \\
{\left[\mathrm{gl}^{-1}\right]}\end{array}$ & $\begin{array}{c}\text { Residual anti-MRSA activity } \\
{[\%]}\end{array}$ \\
\hline Control & 8.00 & 100 & 5.00 & 100 \\
$\mathrm{MgSO}_{4}$ & 5.22 & 75 & 3.66 & 67 \\
$\mathrm{FeSO}_{4}$ & 8.00 & 100 & 5.00 & 93 \\
$\mathrm{MnCl}_{2}$ & 6.22 & 81 & 5.00 & 99 \\
$\mathrm{ZnSO}_{4}$ & 7.30 & 100 & 5.10 & 90 \\
$\mathrm{CuSO}_{4}$ & 6.59 & 90 & 4.82 & 71 \\
$\mathrm{NaCl}_{\mathrm{CaCO}}$ & 4.27 & 65 & 5.5 & 77 \\
$\mathrm{~K}_{2} \mathrm{HPO}_{4}$ & 8.00 & 60 & 4.07 & 60 \\
$\mathrm{KH}_{2} \mathrm{PO}_{4}$ & 4.90 & 71 & 4.31 & 74 \\
\hline
\end{tabular}

S. xanthochromogenus MS-26, as they produced inhibition zones of the highest diameters $(31.5$ and $31.1 \mathrm{~mm}$, respectively). The lowest yield was detected with glucose as the carbon source $(11 \mathrm{~mm})$ - Table 10 . The utilization of glucose, glycerol, and maltose for the growth and the production of anti-MRSA metabolites indicated the existence of an active uptake system for these substrates in $S$. vastus RS-9 and S. xanthochromogenus MS-26 strains. El-Gendy and coworkers (2008b) reported that glucose, starch, or glycerol used as carbon source ensured a high yield of ayamycin antibiotic formation by marine Nocardia sp. ALAA-2000.

\section{The effect of different nitrogen sources} on the production of anti-MRSA metabolites

The data in Table 11 show that the production of anti-MRSA metabolites was significantly affected by the nature of the source of nitrogen added to the culture medium. An organic source of nitrogen in the form of powdered peanuts, followed by yeast extract and peptone, gave the best yield in the production of antiMRSA metabolites by $S$. vastus RS-9, as evidenced by the diameters of inhibition zones, which were 39, 38.3, and $38.1 \mathrm{~mm}$, respectively. Soybean meal stimulated the highest anti-MRSA productivity by $S$. xanthochromogenus MS-26 as the inhibition zone diameter was $41.5 \mathrm{~mm}$ (Table 11). The highest growth levels of RS-9 strain $\left(10 \mathrm{gl}^{-1}\right)$ and MS-26 strain $\left(7.16 \mathrm{gl}^{-1}\right)$ were detected when the growth medium was supplemented with a corn meal extract and a fish meal extract, respectively (Table 11). Our data are in agreement with those ob- tained by Vasavada and coworkers (2006) on S. sannanensis RGT-1, with El-Gendy and coworkers (2008b) on Nocardia sp. ALAA-2000, with Kumar and coworkers (2011) on A. alba, and with Atta and coworkers (2011) on Streptomyces antibioticus AZ-Z710. On the other hand, the production of anti-MRSA metabolites increased by $37.5,32.8,25,7.8$, and $57.1 \%$, when compared to the controls $\left(\mathrm{NaNO}_{3}\right)$, in the cultures of $S$. vastus RS-9 containing valine, arginine, asparagine, lysine, and a mixture of valine and powdered peanut as nitrogen sources, respectively, with $1 \%$ glucose as the carbon source. $\mathrm{Cul}-$ tures containing asparagine, methionine, and a mixture of soybean meal, methionine and asparagine stimulated anti-MRSA productivity in S. xanthochromogenus MS-26 by $1.30,1.35$, and 1.52 -fold, respectively, compared to the controls (Table 11). Positive effects of certain amino acids on the production of secondary metabolites may be due to their direct incorporation in to the chromospheres of bioactive molecules, as reported previously for the enhancement of the biosynthesis of actinomycin D (actD), granaticin, and dimethyltetracycline by Streptomyces chrysomallus, Streptomyces violaceolatus, and Streptomyces aureofaciens Sub-species viridulans, respectively (Mansour et al., 1996).

\section{The effect of metal sources on the production of anti-MRSA metabolites}

The results given in Table 12 indicate that some metal ions play a significant role in the promotion of the production of anti-MRSA metabolites in both tested strains. The anti-MRSA yield from $S$. vastus RS-9 in the 
Table 13. Gas chromatography-mass spectrometry (GC-MS) of volatile compounds identified in S. vastus RS-9 and S. xanthochromogenus MS-26 strains

\begin{tabular}{|c|c|c|c|c|c|}
\hline \multicolumn{3}{|c|}{ S. vastus RS-9 } & \multicolumn{3}{|c|}{ S. xanthochromogenus MS-26 } \\
\hline No. & Compounds & R.A [\%] & No. & Compounds & R.A [\%] \\
\hline 1 & $\mathrm{~N}$-(3-Acetyl phenyl)benzene ethanamide & 10.22 & 1 & Dihydro-2,5-furandione & 0.43 \\
\hline 2 & 1,3-Dinitro-2-methylbenzene & 0.42 & 2 & 1,1,2,2-Tetrachloro-1-fluoroethane & 1.20 \\
\hline 3 & (1,1-dimethylethyl)dimethyl 1-(Phenylmethoxy) silane & 1.45 & 3 & Diethyl butanedioate & 0.86 \\
\hline 4 & Phenol & 2.15 & 4 & 2-Coumaranone & 0.50 \\
\hline 5 & 1,1,1,3,5,5,5-Heptamethyl Trisiloxane & 0.99 & 5 & 1-Amino-2-acetamino-3-fluorobenzene & 2.33 \\
\hline 6 & $\beta$-hydroxy ethylbenzene & 1.59 & 6 & Ethyl N-(2-methyl phenyl)carbamate & 0.33 \\
\hline 7 & 2-Methyl-2-[(2-Methyl-2-propenyl)oxy]-1-propanol & 10.16 & 7 & 2-Hydroxy-2,4,6-cycloheptatrien-1-One & 0.28 \\
\hline 8 & Erythritol & 2.49 & 8 & 2-Hydroxy-2,4,6,-cycloheptatien-1-One & 0.99 \\
\hline 9 & N-Methoxy-N-methylacetamide & 2.15 & 9 & Methyl hexapyranoside & 3.22 \\
\hline 10 & Piperidinone & 1.10 & 10 & $\beta$,D-Methyl Glucopyranoside & 0.58 \\
\hline 11 & A-Piperidinone & 1.29 & 11 & $\beta, \mathrm{D}-$ Methyl Glucopyranoside & 1.11 \\
\hline 12 & 1,7,7-Trimethyl-bicyclo[2-2-1] heptane & 0.16 & 12 & $\alpha$-D-Methyl Glucopyranoside & 2.43 \\
\hline 13 & 1-Amino-2-acetamino-3-fluorobenzene & 3.46 & 13 & $\alpha$-D-Methyl glucopyranoside & 3.61 \\
\hline 14 & Methyl- $\alpha$-D-galactopyranoside & 2.29 & 14 & 2-(Dimethyl hydrazono)-butanol & 2.05 \\
\hline 15 & {$[4,4,8,8-\mathrm{D}(4)]$ Spiro[2.5]Octan-6-One } & 1.88 & 15 & 4,4A, $\beta-5,6,7,7 \mathrm{~A}-\beta-H e x a h y d r o-7, \alpha ́$-methyl cyclopenta[C] Pyran-3-(1H)-one & 4.69 \\
\hline 16 & Ethanedithioamide & 1.14 & 16 & E,E-2,4-decadienol & 1.48 \\
\hline 17 & 2,3-Dihydrothiazolo[3,2-C]pyrimidinium-8-olate & 4.92 & 17 & Octahydro-7a-hydroxy-1H-Inden & 0.86 \\
\hline 18 & 2-Pentyl-3,4-dihydro-2H-pyrane & 1.10 & 18 & 2-Hydroxy-3,5,5-trimethyl-cyclohex-2-enone & 1.17 \\
\hline 19 & (R)-4-Hydroxy-2,6,6-Trimethylcyclohex-2-en-lone & 0.94 & 19 & Palmitic acid methyl ester & 1.66 \\
\hline 20 & 2,5,5-Trimethyl-1',3-cyclohexanedione & 0.96 & 20 & Phenylthio acetaldehyde & 0.34 \\
\hline 21 & 1-butyl-1-methyl-2-propyl cyclopropane & 1.67 & 21 & 1-Methyl-2-acetyl-6-acetoxy-7-methoxy-(1,2,3,4-tetra hydro isoquinoline & 2.03 \\
\hline 22 & Methyl hexadecanoate & 3.85 & 22 & $\beta$-Isobutyl hexahydro pyrrolo[1,2-a] pyrazine-1,4-dione & 1.61 \\
\hline 23 & 3-Isobutyl hexahydropyrrolo[1,2- $\alpha]$ pyrazine-1,4-dione & 2.13 & 23 & n-Hexadecanoic acid & 22.13 \\
\hline 24 & Hexadecanoic acid & 9.87 & 24 & Ethyl hexadecanoate & 2.62 \\
\hline 25 & Ethyl palmitate & 2.54 & 25 & Methyl (8E, 11E)-8,11-octadecadienoate & 4.56 \\
\hline 26 & Palmitic acid & 1.21 & 26 & Methyl(9Z)-9-octadecenoate & 3.03 \\
\hline 27 & Linoleic acid & 7.75 & 27 & Methyl Stearate & 0.81 \\
\hline 28 & Elaidic acid & 4.88 & 28 & Cis-9, Cis-12-Linoleic Acid & 17.85 \\
\hline 29 & Stearic acid methylester & 1.10 & 29 & (9Z,12Z)-Octadecadienoic Acid & 2.15 \\
\hline 30 & $(Z, Z)-9,12$ octa decadienoic acid & 18.01 & 30 & Cis, Cis-Linoleic Acid & 2.03 \\
\hline 31 & Cis, Cis Linoleic acid & 0.93 & 31 & 2-Thioxo octahydro-4H-Cyclopenta[d]pyrimidine-4-one & 0.25 \\
\hline 32 & Ethyl-15-methylhectadecanoate & 0.57 & 32 & 4H-[1,2,5]Oxadiazolo[3,4-D] Pyrimidine-5,7-dione-1-oxide & 2.30 \\
\hline 33 & 9E, 12E-Octadecadienoic acid & 0.13 & 33 & 3-Benzyl hexahydro pyrolo[1,2-A] Pyrazine-1,4-dione & 0.65 \\
\hline 34 & Bi S (2-ethyl hexyl) phthalate & 1.13 & 34 & N-(4-methoxyphenyl)-2-oxocyclopropaneoctanal-2-octyl-1-Oxaspiro[4-4]nonane-4-carboxamide & 0.18 \\
\hline 35 & 2,6-Dimethoxy phenyl acetate & 2.09 & 35 & 4-(dimethyl amino)-1,4,4d,5,12,12a-hexahydro-3,10,11,12a-tetrahydroxy-6-methyl-1,12-dioxo-, & 10.27 \\
\hline \multirow[t]{4}{*}{36} & 5-nitro-2-propoxy benzenamine & 2.03 & & {$[4 \mathrm{~S}-(4-\alpha, 4 \mathrm{a}, \mathrm{al}]-(2$-naphthacene carboxamide) } & \\
\hline & & & 36 & Bis(2-ethylhexyl)phthalate & 11.0 \\
\hline & & & 37 & 1-(4-Chlorophenyl)-piperazine & 13.40 \\
\hline & & & 38 & 3,5-Dimethoxy phenyl acetate & 0.01 \\
\hline
\end{tabular}




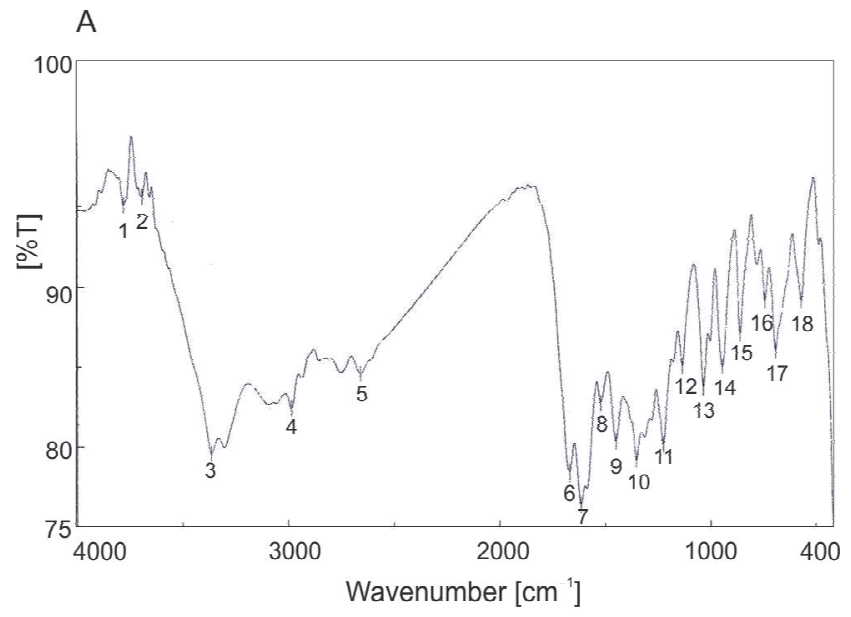

B

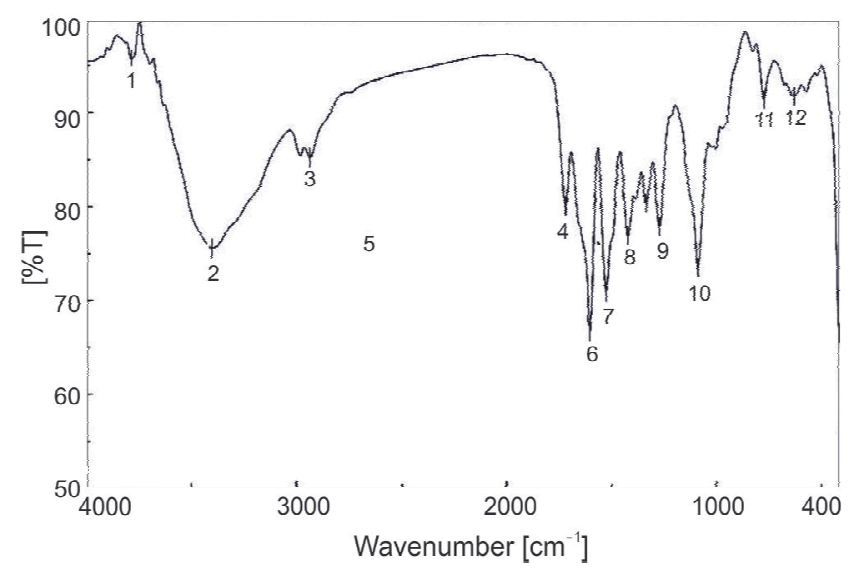

Fig. 3. IR spectrum of compounds A and B produced by S. xanthochromogenus MS-26 strain

absence of $\mathrm{MgSO}_{4}, \mathrm{MnCl}_{2}, \mathrm{CuSO}_{4}, \mathrm{NaCl}, \mathrm{CaCO}_{3}, \mathrm{~K}_{2} \mathrm{HPO}_{4}$, or $\mathrm{KH}_{2} \mathrm{PO}_{4}$ (individually from the medium) was decreased by $25,19,10,35,40,29$, and $11 \%$, but in $S$. xanthochromogenus MS-26 in the absence of these metal ions the reduction equaled to $33,1,16,29,23,40$, and $26 \%$, respectively (Table 12). Moreover, $\mathrm{FeSO}_{4}$ and $\mathrm{ZnSO}_{4}$ had no effect on the production of anti-MRSA metabolites in RS-9 strain, but they decreased their production in MS26 strain by 7 and $10 \%$, respectively. These results are in agreement with those obtained by Kishimoto and coworkers (1996), who were testing the influence of metal ions on the growth of Streptoverticillium rimofaciens and mildiomycin antibiotic productivity as well as granaticin production in S. violaceolatus (Mansour et al., 1996).

\section{Characterization of volatile compounds produced by $S$. vastus $R S-9$ and $S$. xanthochromogenus MS-26 using the $G C / M S$ analysis}

The GC/MS analysis showed that the active particles in $S$. vastus RS-9 strains such as $(Z, Z)-9,12-)$ ctadeca- dienoic acid (18.01\%), N-(3-acetyl phenyl)benzene ethanamide (10.22\%), 2-methyl-2-[(2-methyl-2-propenyl)oxy]1-propanol (10.16\%), hexadecanoic acid (9.87\%), linoleic acid (7.75\%), 2,3-dihydrothiazolo[3,2-C]pyrimidinium-8olate (4.92\%), elaidic acid (4.88\%), and methyl hexadecanoate $(3.85 \%)$ as the major compounds (Table 13$)$. On the other hand, volatile organic compounds (VOCs) in S. xanthochromogenus MS-26 strains were found to be nhexadecanoic acid (22.13\%), cis-12-linoleic acid (17.85\%), Bis(2-ethylhexyl)phthalate (11.0\%), cis-9,4-(dimethyl amino)-1,4,4d,5,12,12a-hexahydro-3,10,11,12a-tetrahydroxy-6-

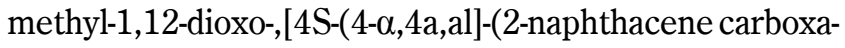
mide) (10.27\%), 4,4A, $\beta-5,6,7,7 \mathrm{~A}-\beta$-hexahydro-7,ó-methyl cyclopenta[C]pyran-3-(1H)-one (4.69\%), and methyl (8E, 11E)-8,11-octadecadienoate (4.56\%) - Table 13. Different bioactive substances were detected in the extract of different marine endophytic Streptomycetes and fungi, for example, prodigiosin-like pigments from endophytic marine Streptomyces species, a small molecule with antiMRSA activity from Streptomyces sp. PVRK-1, two metabolites (KGG32-A and KGG32-B) from Streptomyces sp. KGG32, bioactive benzopyrone derivatives from Streptomyces, ayamycin antibiotic from Nocardia sp. ALAA 2000, anti-MRSA/VRE antibiotic laidlomycin from Streptomyces sp. CS684, and meroparamycin from Streptomyces sp. MAR01 (El-Bondkly et al., 2012b; Kannan et al., 2011; Oskay 2011; El-Gendy et al., 2008a and b; Yoo et al., 2007; El-Naggar et al., 2006).

\section{Structure elucidation of pure compounds $A$ and $B$ isolated from $S$. xanthochromogenus MS-26 strain}

Based on the detailed spectral data of pure compounds (A and B) (Fig. 3, Fig. 4, and Fig. 5) and comparing the data with the reports in the literature, compound A is elucidated as 1-Methyle-2-acetyle-6-acetoxy-7methoxy-(1,2,3,4-tetrahydroisoquinoline) with the chemical formula $\mathrm{C}_{15} \mathrm{H}_{19} \mathrm{NO}_{4}$ and molecular weight $277 \mathrm{Da}$. On the other hand, compound $\mathrm{B}$ is 4-(dimethylamino)1,4,4d,5,12,12a-hexahydro-3,10,11,12a-tetrahydroxy-6methyl1-1,12-dioxo-,[4S-(4- $\alpha, 4 \mathrm{a}, \mathrm{a} 1]$-(2-naphthacene carboxamide), which is a well-known antibiotic anhydrotetracycline with the chemical formula $\mathrm{C}_{22} \mathrm{H}_{22} \mathrm{~N}_{2} \mathrm{O}_{7}$ and molecular weight $426 \mathrm{Da}$ (Fig. 6). A comparison of compound $A$ with structure related to isoquinolines resulted in different compounds, which have an identical basic structure, such as the anhalonines with the molecular formula of $\mathrm{C}_{12} \mathrm{H}_{15} \mathrm{NO}_{3}$ and molecular weight equal to 

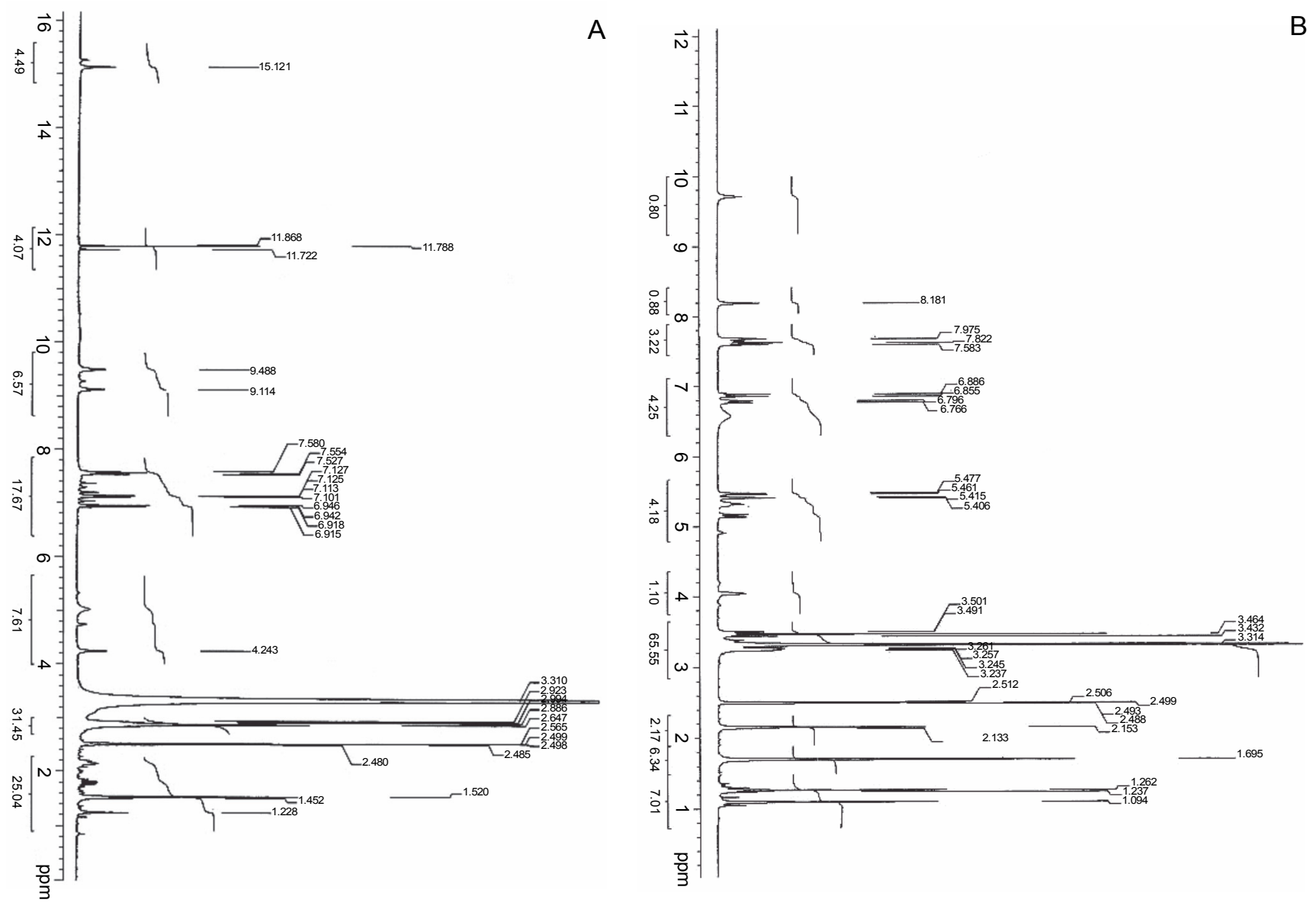

Fig. 4. HNMR spectrum of compounds A and B produced by $S$. xanthochromogenus MS-26 strain

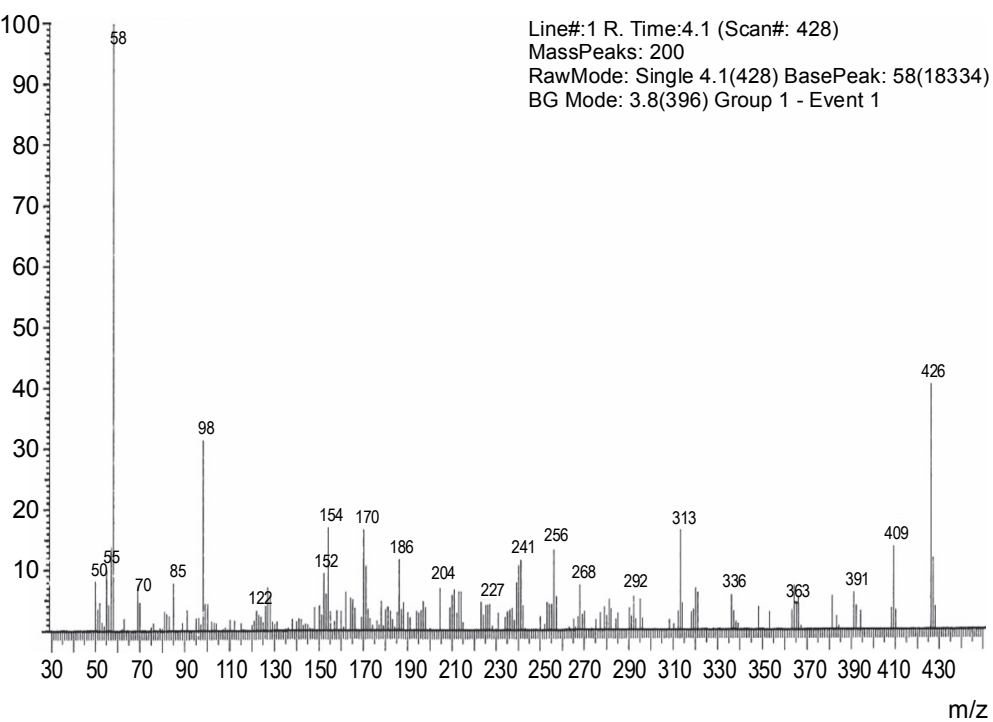

Fig. 5. Mass spectrum of the highly active compound B produced by $S$. xanthochromogenus MS-26 strain 
Table 14. Minimum inhibitory concentration (MIC) and minimum bactericidal concentration (MBC) values of the pure compounds (A and B) isolated from $S$. xanthochromogenus MS-26 against clinical isolates

\begin{tabular}{|c|c|c|c|c|}
\hline \multirow{2}{*}{ Clinical isolates } & \multicolumn{2}{|c|}{ Compound A $\left[\mu \mathrm{gml}^{-1}\right]$} & \multicolumn{2}{|c|}{ Compound $\mathrm{B}\left[\mu \mathrm{gml}^{-1}\right]$} \\
\hline & MIC & $\mathrm{MBC}$ & MIC & $\mathrm{MBC}$ \\
\hline \multicolumn{5}{|l|}{ MRSA strains } \\
\hline MRSA-D1 & 1024 & 1100 & 16 & 20 \\
\hline MRSA-D2 & 1024 & 1050 & 32 & 32 \\
\hline MRSA-D3 & 256 & 300 & 32 & 40 \\
\hline MRSA-D4 & 1024 & 1050 & 64 & 68 \\
\hline MRSA-D6 & 32 & 40 & 8 & 8 \\
\hline MRSA-D7 & 512 & 562 & 32 & 36 \\
\hline MRSA-D8 & 256 & 264 & 32 & 32 \\
\hline MRSA-D9 & 16 & 16 & 32 & 36 \\
\hline MRSA-D10 & 32 & 40 & 32 & 40 \\
\hline MRSA-D11 & 1024 & 1250 & 128 & 128 \\
\hline MRSA-D12 & 16 & 24 & 8 & 8 \\
\hline MRSA-D13 & 128 & 134 & 16 & 24 \\
\hline MRSA-D14 & 256 & 264 & 64 & 68 \\
\hline MRSA-AS16 & 512 & 520 & 16 & 16 \\
\hline MRSA-AS17 & 512 & 520 & 32 & 40 \\
\hline MRSA-AS18 & 512 & 528 & 32 & 36 \\
\hline MRSA-AS19 & 1024 & 1100 & 16 & 24 \\
\hline MRSA-AS20 & 1024 & 1030 & 32 & 36 \\
\hline \multicolumn{5}{|l|}{ VI-MRCoNS strains } \\
\hline CoNS-D5 & 128 & 150 & 32 & 40 \\
\hline CoNS-AS15 & 128 & 128 & 4 & 8 \\
\hline
\end{tabular}

Table 15. Cytotoxic activity of the pure compounds (A and B) against different cancer cell lines

\begin{tabular}{|c|c|c|c|c|c|c|c|c|c|c|}
\hline \multirow{3}{*}{ Cell line* } & \multicolumn{10}{|c|}{ Cytotoxic activity (\%) } \\
\hline & \multicolumn{5}{|c|}{ Compound A } & \multicolumn{5}{|c|}{ Compound B } \\
\hline & $\begin{array}{l}1.25 \\
\text { PPM }\end{array}$ & $\begin{array}{l}2.50 \\
\text { PPM }\end{array}$ & $\begin{array}{l}5.00 \\
\text { PPM }\end{array}$ & $\begin{array}{l}10.00 \\
\text { PPM }\end{array}$ & $\mathrm{IC}_{50}$ & $\begin{array}{l}1.25 \\
\text { PPM }\end{array}$ & $\begin{array}{l}2.50 \\
\text { PPM }\end{array}$ & $\begin{array}{c}5.00 \\
\text { PPM }\end{array}$ & $\begin{array}{l}10.00 \\
\text { PPM }\end{array}$ & $\mathrm{IC}_{50}$ \\
\hline A-549 & 2.3 & 0.9 & 9.2 & 16.1 & $>50 \%$ & 5.7 & 0.4 & 0.0 & 0.82 & $>50 \%$ \\
\hline HCT-116 & 12 & 15.9 & 21.3 & 28 & $>50 \%$ & 6.5 & 14 & 21.6 & 23.1 & $32.2 \mu \mathrm{g} / \mathrm{ml}$ \\
\hline HepG-2 & 21.2 & 32 & 35.8 & 43.7 & $20 \mu \mathrm{g} / \mathrm{ml}$ & 8.6 & 14.2 & 15.6 & 15.9 & $>50 \%$ \\
\hline
\end{tabular}

*A-549 - lung cancer cell line, HCT-116 - colon cancer cell line, HepG-2 - liver cancer cell line, IC50 - the concentration that gave $50 \%$ death of the tumor cells

257.7131 Da, Chaetoindicins A-C isolated from the fungus Chaetomium indicum with the molecular formula $\mathrm{C}_{16} \mathrm{H}_{19} \mathrm{NO}_{4}$ and fusarimine from the endophytic fungus Fusarium sp. LN12 (Li et al., 2006; Yang et al., 2012), but the structure of compound B was identical to that of a anhydrotetracycline compound. Both isoquinoline and anhydrotetracycline have been reported to exert various pharmacological activities such as anticancer, antihypertensive, antimicrobial, cerebral vasodilation, etc. (Li et al., 2006; Yang et al., 2012). Lemonomycin, for example, is an isoquinoline compound isolated from Streptomyces candidus with a potent activity against Bacillus subtilis,
S. aureus, MRSA, and vancomycin-resistant Enterococci as well as anticancer activity against colon cancer cell lines, HCT116 (El-Gendy et al., 2008a). Coumarin antibiotics isolated from Streptomycetes proved to be antimicrobial against a variety of microorganisms as reported by El-Gendy and coworkers (2008a).

\section{Determination of the MIC and MBC of compounds $A$ and $B$ against MRSA and CoNS isolates}

Compound $\mathrm{B}$ possessed a higher activity against all MRSA and VI-MRCoNS isolates tested than the compound $\mathrm{A}$, with $\mathrm{MIC}$ ranging from 4 to $8 \mu \mathrm{gml}^{-1}$ and $\mathrm{MBC}$ 
A

B

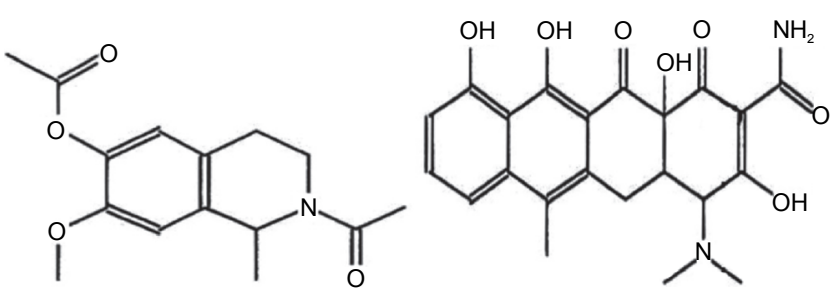

Fig. 6. The structure of compound (A) [1-Methyl-2-acetyl-6-acetoxy-7-methoxy-(1,2,3,4-tetrahydro isoquinoline)] and compound (B) [4-(dimethyl amino)-1,4,4d,5,12,12a-hexahydro-3,10,11,12aTetrahydroxy-6-methyl-1,12-dioxo-,[4S-(4- $\alpha, 4 \mathrm{a}$, al]-(2-naphthacene carboxamide)] produced by $S$. xanthochromogenus MS-26 strain

ranging from 8 to $128 \mu \mathrm{gml}^{-1}$. Compound A was characterized by MIC in a range between 16 and $1024 \mathrm{gmm}^{-1}$, and $\mathrm{MBC}$ values ranging from 16 to $1250 \mu^{\mathrm{gml}}{ }^{-1}$ (Table 14). Interestingly, both compounds had a potent inhibitory activity against VI-MRCoNS-D5 isolates, which were isolated from a leukemia patient OR from leukemia patients with intermediate resistance to vancomycin only, and against the multidrug resistant MRSA isolates (MRSA-D6, MRSA-D9, MRSA-D12, MRSA-D13 and MRSA-D14) that were resistant to all antibiotics except two, one of which was vancomycin. In previous studies the effective anti-MRSA compounds such as anthranilamides, SBR-22, CSU-1, and KGG32 (A and B were isolated from Streptomyces sp. B7747, Streptomyces BT408, Streptomyces sp. CS684, and Streptomyces sp. KGG32 with MIC values equal to 107.0, 64.0, 1.0, and (6.25 and 12.5) $\mu \mathrm{gml}^{-1}$, respectively (Biabani et al., 1998; Sujatha et al., 2005; Yoo et al., 2007; Oskay, 2011).

\section{Anticancer activity of compounds $A$ and $B$}

The anticancer activities of compounds $A$ and $B$ on liver (HEPG-2), colon (HCT-116), and lung (A-549) cancer cell lines were evaluated based on the MTT cell viability assay. The data in Table 15 clearly indicate that liver cancer HEPG-2, colon cancer HCT-116, and lung cancer A-549 cell lines were more sensitive to compound $\mathrm{A}$ than to compound $\mathrm{B}$. Compound $\mathrm{A}$ at $10 \mathrm{ppm}$ showed cytotoxic activity of $16.1,28.0$, and $43.7 \%$ against A-549, HCT-116, and HEPG-2, respectively; 10 ppm of compound $\mathrm{B}$ inhibited their viability by $0.82,23.1$, and $15.9 \%$, respectively. Moreover, compound A (belonging to the group of isoquinolines antibiotics) exhibited a considerable cytotoxic activity on liver cancer cell line as
$\mathrm{IC}_{50}$ value of $20 \mu \mathrm{gml}^{-1}$, while compound $\mathrm{B}$, which belongs to the group of coumarin antibiotics, showed a significant cytotoxic activity on colon cancer cell line with $\mathrm{IC}_{50} 32.2 \mu \mathrm{gml}^{-1}$. These findings indicate that endophytic Actinomycetes are reservoirs of various bioactive metabolites that may serve as potential anticancer/antimicrobial drugs for patients in critical health conditions (ICU), immunocompromised patients (such as cancer patients) and those with multidrug MRSA infections. The results of this work are consistent with the data on the antitumor activities of bioactive metabolites derived from marine Actinomycetes such as Streptomyces avidinii strain SU4, reported by Sudha and Selvam (2012).

\section{Conclusions}

In Egypt, marine ecosystems proved to be important sources of different marine endophytic Streptomyces species that produce various interesting bioactive metabolites against bacteria causing infectious diseases and against cancer, identified as methicillin-resistant $S$. aureus. Two pure compounds, 1-Methyl-2-acetyl-6-acetoxy7-Methoxy-(1,2,3,4-Tetrahydro-isoquinoline) and 4-(dimethyl amino)-1,4,4d,5,12,12a-hexahydro-3,10,11,12a-Tetrahydroxy-6-Methyl-1,12-dioxo-,[4S-(4- $\alpha, 4 \mathrm{a}$,al]-(2-naphthacene carboxamide) isolated from $S$. xanthochromogenus MS-26 showed a potent anti-MRSA activity against all tested clinical MRSA and CoNS isolates with MIC ranging from 16 to 1024 and from 4 to $128 \mu \mathrm{gml}^{-1}$, respectively, while MBC ranging from 16 to 1024 and from 8 to $128 \mathrm{mgml}^{-1}$, respectively, as well as an anticancer activity against colon ( HCT-16), liver (HepG-2), and lung cancer cell lines.

\section{Conflicts of interest}

All authors have declared that there have been no conflicts of interest.

\section{Contributors}

El-Gendy M.M.A.A., Mohamed Z.K., Hekal N.Z., Ali F.M., and Yousef A.E.M. designed the study, preformed the experiments, managed literature searches, data analyses, and wrote the manuscript. All the authors read and approved the final manuscript.

\section{Ethical approval}

Not required. 


\section{References}

Ahmed M.M.A., Nageh Abo Dahab F., Taher Taha M., Fareed Hassan S.M. (2015) Production, purification and characterization of L-asparaginase from marine endophytic Aspergillus sp. ALAA-2000 under submerged and solid state fermentation. J. Microb. Biochem. Technol. 7(3): 165-172.

Atta H.M., El-Sehrawi M.H., Awny N.M., El-Mesady N.I. (2011) Microbiological studies on cultural, physiological characteristics and antimicrobial activities of Streptomyces cyaneus AZ-13Zc. Researcher 3(2): 80-90.

Awadalla I.H., Khalil A.I., Bassim H.H., Ahmed M.N., Wahba M.L. (2010) Molecular typing of methicillin resistant Staphylococcus aureus isolates at Ain Shams University Hospital, Egypt. Afr. J. Microbiol. Res. 4(15): 1639-1646.

Baddour M.M., Abuelkheir M.M., Fatani A.J. (2006) Trends in antibiotic susceptibility patterns and epidemiology of MRSA isolates from several hospitals in Riyadh, Saudi Arabia. Ann. Clin. Microbiol. Antimicrobials 5: 30-40.

Biabani M.A., Baake M., Lovisetto B., Latsch H., Helmke E., Weyland H. (1998) Anthranilamides: new antimicroalgal active substances from marine Streptomyces sp. J. Antibiot. 51(3): 333-340.

Butte W. (1983) Rapid method for the determination of fatty acid profiles from fats and oils using trimethylsulphonium hydroxide for transesterification. J. Chromatogr. 261: 142-145.

Cappuccino J.G., Sherman N. (1999) Microbiology: A Laboratory Manual, $4^{\text {th }}$ Ed., pp: 199-204, Addison Wesley Longman, Inc. Harlow, England.

Cheesbrough M. (2000) District laboratory practice in tropical countries (part 2). pp. 62-70, Cambridge University Press, UK.

Cherkaoui A., Renzi G., Francois P., Schrenzel G. (2007) Comparison of four chromogenic media for culture based screening of methicillin resistant Staphylococcus aureus. J. Med. Microiol. 56: 500-503.

Collee J.G., Duguid J.P., Fraser A.G., Marmion B.P. (1989) The Staphylococci. [in:] Machie and McCartney Practical Medical Microbiology $30^{\text {th }}$ edition, p. 303-315. Churchill Livingstone, Edinburgh-London and New York.

Collee J.G., Miles R.S., Watt B. (1996) Tests for the identification of bacteria. [in:] Machie and McCarteny Practical Medical Microbiology, Collee J.G., Fraser A.G., Marmion B.P., Simmons A., $14^{\text {th }}$ edition, p. 131-150, Churchill Livingstone.

Disalvo J.W. (1958) Deoxyribonuclease and coagulase activity of Micrococci. Med. Tech. Bull. US Armed Forces Med. J. 9: 191.

El-Bondkly A.M., El-Gendy M.M.A., Wiese J., Imhoff J.F. (2012a) Phylogenetic diversity and antimicrobial activities of culturable endophytic actinobacteria isolated from different Egyptian marine sponges and soft corals. Aust. J. Basic. Appl. Sci. 6(4): 25-33.

El-Bondkly A.M., El-Gendy M.M.A., Bassyouni R.H. (2012b) Overproduction and biological activity of prodigiosin-like pigments from recombinant fusant of endophytic marine
Streptomyces species. Antonie Van Leeuwenhoek 102(4): 719-734.

El-Gendy M.M.A., Shaaban M., El-Bondkly A.M., Shaaban A.K. (2008a) Bioactive benzopyrone derivatives from new recombinant fusant of marine Streptomyces. Appl. Biochem. Biotechnol. 150: 85-96.

El-Gendy M.M.A., Hawas U.W., Jaspars M. (2008b) Novel bioactive metabolites from a marine derived bacterium Nocardia sp. ALAA 2000. J. Antibiot. 61(6): 379-386.

El-Naggar M.Y., El-Assar S.A., Abdul-Gawad S.M. (2006) Meroparamycin production by newly isolated Streptomyces sp. strain MAR01: Taxonomy, fermentation, purification and structural elucidation. J. Microbiol. 432-438.

Hasegawa T., Takizawa M., Tanida S. (1983) A rapid analysis for chemical grouping of aerobic Actinomycetes. J. Gen. Appl. Microbiol. 29: 319-322.

Hass D., Defago G. (2005) Biological control of soil borne pathogens by fluorescent Pseudomonas. Nat. Rev. Microbiol. 3(4): 369 -372.

Kämpfer P., Kroppenstedt R.M., Dott W. (1991) A numerical classification of the genera Streptomyces and Streptoverticillium using miniaturized physiological tests. J. Gen. Microbiol. 137: 1831-1891.

Kannan R.R., Iniyan A.M., Prakash V.S.G. (2011) Isolation of a small molecule with anti-MRSA activity from a mangrove symbiont Streptomyces sp. PVRK-1 and its biomedical studies in Zebrafish embryos. Asian Pac. J. Trop. Biomed. 1(5): 341-347.

Kawato M., Shinobu R. (1959) On Streptomyces herbaricolor sp. nov., supplement a single technique for microscopical observation. Mem. Osaka Unit. Lib. Arts Educ. B Nat. Sci. 8: 114-119.

Kishimoto K., Park Y.S., Okabe M., Akiyama S. (1996) Effect of phosphate ion on mildiomycin production by Streptoverticillium rimofaciens. J. Antibiot. 49: 775-780.

Kloos W.E., Lambe D.W. (1991) Staphylococcus. [in:] Manual of Clinical Microbiology. Ed. Balows A., Hasusler W.J., Hermann K.L. Washington DC, American Society for Microbiology: 222-230.

Kumar V.R.R.D., Yugandar M.N., Reddy S.R.D. (2011) Screening of antagonistic Actinomycetes: optimization of process parameters for the production of novel antibiotic by Amycolatopsis alba var. nov. DVRD4. J. Microbial Biochem. Technol. 3: 92-98.

Lairrull L.I., Fisher J..F, Mobashery S. (2009) Molecular basis and phenotype of methicillin resistance in Staphylococcus aureus and insights into new $\beta$-lactams that meet the challenge. Antimicrob. Agents Chemother. 53(10): 4051-4063.

Lavermicocca P., Valerio F., Visconti A. (2003) Antifungal activity of phenyl lactic acid against molds isolated from bakery products. Appl. Environ. Microbiol. 69: 634-640.

Lechevalier M.P., De-Bievre C., Lechevalier H.A. (1977) Chemotaxonomy of aerobic Actinomycetes: phospholipid composition. Biochem. Syst. Ecol. 5: 249-260.

Li Z. (2009) Advances in marine microbial symbionts in the China Sea and related pharmaceutical metabolites. Review Mar. Drugs 7: 113-129. 
Li G.-Y., Li B.-G., Tao Y., Liu G.-Y., Zhang G.-L. (2006) Chaetoindicins $A-C$, three isoquinoline alkaloids from the fungus Chaetomium indicum. Organic. Lett. 8: 36133615.

Mandel M., Marmur J. (1968) Use of ultraviolet absorbance temperature profile for determining the guanine plus cytosine content of DNA. Methods Enzymol. 12B: 195-206.

Mansour F.A., El-Shirbiny S.A., El-Metwaly N.A. (1996) Demethyltetracycline biosynthesis by Streptomyces aureofaciens Sub-species viridulans as influenced by medium composition. Egyptian J. Microbiol. 31: 221-235.

Minnikin D.E., Patel P.V., Alshamaony L., Goodfellow M. (1977) Polar lipid composition in the classification of Streptomyces and related bacteria. Int. J. Syst. Bacteriol. 27: 104-117.

Mosmann T. (1983) Rapid colorimetric assays for cellular growth and survival: Application to proliferation and cytotoxicity assay. J. Immunol. Methods 65: 55-63.

Najjuka F.C., Kateete D.P., Kimani C.N., Katabazi F.A., Okeng A., Okee M.S., Nanteza A., Joloba M.L. (2010) Identification of Staphylococcus aureus: DNase and mannitol salt agar improve the efficiency of the tube coagulase test. Ann. Clin. Microbiol. Antimicrobials 9: 23.

Nithyanand P., Pandian S.K. (2009) Phylogenetic characterization of culturable bacterial diversity associated with the mucus and tissue of the coral Acropora digitifera from the Gulf of Mannar. FEMS Microbiol. Ecol. 69: 384-394.

Oskay M. (2011) Isolation and purification of two metabolites (KGG32-A \& KGG32-B) from a soil bacterium, Streptomyces sp. KGG32. Int. J. Agric. Biol. 13(3): 369-374.

Padalkar R.R., Peshwe S.A. (2013) Isolation and characterization of new anti-MRSA producing Actinomycete. Indian J. Appl. Res. 3(6): 433-435.

Procop G.W., Shrestha N.K., Tuohy M.J., Hall G.S., Isada C.M. (2002) Rapid identification of Staphylococcus aureus and the mecA gene from BacT/ALERT blood culture bottles by using the light cycler system. J. Clin. Microbiol. 40(7): 2659-2661.

Sadaka S.M., El-Ghazzawy E.F., Harfoush R.A., Meheissen M.A. (2009) Evaluation of different methods for the rapid diagnosis of methicillin - resistance in Staphylococcus aureus. Afr. J. microbial Res. 3(2): 49-55.

Selva E., Gastaldo L., Saddler G., Toppo G., Ferrari P., Carniti G. (1996) Goldstein B.: Antibiotics A21459 A and B, new inhibitors of bacterial protein synthesis. 1-Taxonomy, isolation and characterization. J. Antibiot. 49(2): 145-149.

Sharaf H., Badawi D., El-Kinawy N. (2006) Simultaneous identification, detection of $S$. aurues and methicillin resistnce by PCR amplification of FemB and mec A. Egypt J. Med. Lab. Sci. 15(2): 1-10.

Shirling E.B., Gottlieb D. (1966) Methods for characterization of Streptomyces species. Int. J. Syst. Bacteriol. 16: 313-340.

Sudha S., Selvam M.M. (2012) Characterization of cytotoxic compound from marine sediment derived actinomycete Streptomyces avidinii strain SU4. Asian Pac. J. Trop. Biomed. 2(10): 770-773.
Sujatha P., Bapiraju K.V., Ramana T. (2005) Studies on a new marine Streptomycetes BT-408 producing polyketide antibiotic SBR-22 effective against methicillin resistant Staphylococcus aureus. Microbiol. Res. 160(2): 119-126.

Szabo I.M., Marton M., Buti I., Fermandez C. (1975) A diagnostic key for the identification of species of Streptomyces and Streptoverticillium included in the International Streptomyces Project. Acta Bot. Acad. Sci. Hung. 21: 387-418.

Taechowisan T., Peberdy J.F., Lumyong S. (2003) Isolation of endophytic Actinomycetes from selected plants and their antifungal activity. World J. Microbiol. Biotechnol. 19: 381-385.

Thumar J.T., Dhulia K., Singh S.P. (2010) Isolation and partial purification of an antimicrobial agent from halotolerant alkaliphilic Streptomyces aburaviensis strain Kut-8. World J. Microbiol. Biotechnol. 26: 2081-2087.

Udo E.E., Al-Sweih N., Dhar R., Dimitrov T.S., Mokaddas E.M., Johny M., Al-Obaid I.A., Gomaa H.H., Mobasher L.A., Rotimi V.O., Al-Asar A. (2008) Surveillance of antibacterial resistance in Staphylococcus aureus isolated in Kuwaiti hospitals. Med. Princ. Pract. 17: 71-75.

Valli S., Suvathi S.S., Aysha O.S., Nirmala P., Vinoth K.P., Reena A. (2012) Antimicrobial potential of Actinomycetes species isolated from marine environment. Asian Pac. J. Trop. Biomed. 2(6): 469-473.

Vasavada S.H., Thumar J.T., Singh S.P. (2006) Secretion of a potent antibiotic by salt-tolerance and alkaliphilic actinomycete Streptomyces sannanensis strain RJT-1. Saurashtra University, Rajkot 360 005, India. 10: 25.

WilliamsS.T., Goodfellow M., Alderson G., Wellington E.M.H., Sneath P.H.A., Sackin M.J. (1983) Numerical classification of Streptomyces and related genera. J. Gen. Microbiol. 129: 1743-1813.

Williams S.T., Goodfellow M., Alderson G. (1989) Bergey's manual of systematic bacteriology. Williams S.T., Sharpe M.E., Holt J.G. (Eds.), The Williams and Wilkins Co., Baltimore, USA.

Yang S.-X., Xiao J., Laatsch H., Holstein J.J., Dittrich B., Zhang Q. (2012) Fusarimine, a novel polyketide isoquinoline alkaloid from the endophytic fungus Fusarium sp. LN12 isolated from Melia azedarach. Tetrahedron Lett. 53: 6372-6375.

Yoo J.C., Kim J.H., Ha J.W., Park N.S., Sohng J.K., Lee J.W., Park S.C., Kim M.S., Seong C.N. (2007) Production and biological activity of laidlomycin, anti-MRSA/VRE antibiotic from Streptomyces sp. CS684. J. Microbiol. 45(1): 6-10.

Zaki S.A., Shoala A.Y., El-Ghazzawy E.F., Hussein A.S. (1989) Antibiotic sensitivity pattern of hospital strains of Staphylococcus aureus. M.Sc. Alexandria University, Egypt.

Zhou Z.H., Liu Z.H., Qian Y.D., Kim S.B., Goodfellow M. (1998) Saccharopolyspora spinosporotrichia sp. nov., a novel Actinomycete from soil. Int. J. Syst. Bacteriol. 48: 53-58. 